\title{
过氧化腿在氧化反应中的应用研究进展
}

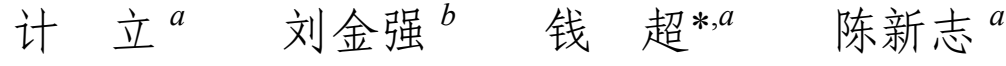 \\ ( ${ }^{a}$ 浙江大学化学工程与生物工程学系 杭州 310027) \\ ( ${ }^{b}$ 洛阳师范学院化学化工学院 洛阳 471022)
}

\begin{abstract}
摘要 氧化反应是重要的有机合成单元反应, 常采用 $\mathrm{H}_{2} \mathrm{O}_{2}$ 或过氧酸等为氧化剂. 近年来, 过氧化腿作为一种清洁环保 的固态 $\mathrm{H}_{2} \mathrm{O}_{2}$, 以其性质稳定、 $\mathrm{H}_{2} \mathrm{O}_{2}$ 含量高 $(36.2 \%$ )且释放可控等优点, 在众多氧化反应中得到了广泛应用. 对过氧化腿 在氧化反应中的应用进行了总结和概述, 重点介绍了环氧化、Baeyer-Villiger 氧化、 $N$-氧化、硫梄氧化成砜和亚砜、氧 化卤化等反应.
\end{abstract}

关键词＼cjkstart过氧化腿; 环氧化; Baeyer-Villiger 氧化; $N$-氧化; 砜; 亚砜; 氧化卤化

\section{Advances in the Application of Urea-Hydrogen Peroxide to Oxidation Reactions}

\author{
$\mathrm{Ji} \mathrm{Li}^{a} \quad$ Liu, Jinqiang ${ }^{b} \quad$ Qian, Chao $^{*, a} \quad$ Chen, Xinzhi $^{a}$ \\ ( ${ }^{a}$ Department of Chemical and Biological Engineering, Zhejiang University, Hangzhou 310027) \\ ( ${ }^{b}$ College of Chemistry and Chemical Engineering, Luoyang Normal University, Luoyang 471022)
}

\begin{abstract}
Oxidation reactions play important roles in organic synthesis, where hydrogen peroxide and peroxy acids are always employed as oxidants. Urea-hydrogen peroxide (UHP) is recently utilized in numerous oxidation reactions as environment-friendly solidified hydrogen peroxide, due to its advantages such as stable at room temperature, high hydrogen peroxide content $(36.2 \%)$ and the potential for releasing it in a controlled manner. Several applications of UHP to organic synthesis are summarized according to reaction type, especially for epoxidation, Baeyer-Villiger oxidation, $\mathrm{N}$-oxidation, oxidation of sulfides to sulfoxides and sulfones, oxidative-halogenation and so on.

Keywords urea-hydrogen peroxide (UHP); epoxidation; Baeyer-Villiger oxidation; $N$-oxidation; sulfones; sulfoxides; oxidative-halogenation
\end{abstract}

环境友好与绿色氧化技术是当前化学研究与开发 的热点. 在众多氧化剂中, $\mathrm{H}_{2} \mathrm{O}_{2}$ 水溶液是一种价廉且环 境友好的绿色氧化剂 ${ }^{[1]}$. 由于大多数有机溶剂与水不互 溶, 因此, 将 $\mathrm{H}_{2} \mathrm{O}_{2}$ 水溶液用于有机物的氧化反应时, 反 应常在两相体系中进行. 常用的 $30 \% \mathrm{H}_{2} \mathrm{O}_{2}$ 水溶液浓度 较小、氧化能力中等、氧化效率低, 常需要加入活化物 质以提高其反应效率 ${ }^{[2]}$, 高浓度 $\mathrm{H}_{2} \mathrm{O}_{2}$ 溶液极易爆炸 ${ }^{[3]}$; 同时 $\mathrm{H}_{2} \mathrm{O}_{2}$ 稳定性较差, 光照 ${ }^{[4]}$ 与金属离子等均可致其分 解, 给试剂的运输和保存带来了困难. $\mathrm{H}_{2} \mathrm{O}_{2}$ 固态化是解 决上述问题的有效途径, 常见的 $\mathrm{H}_{2} \mathrm{O}_{2}$ 固态化形式有过 嗍酸钠 $\left(\mathrm{NaBO}_{3} \bullet n \mathrm{H}_{2} \mathrm{O}, n=1 \sim 4, \mathbf{1}\right)^{[5 \sim 7]}$ 、过碳酸钠 $\left(2 \mathrm{Na}_{2} \mathrm{CO}_{3} \cdot 3 \mathrm{H}_{2} \mathrm{O}_{2}, 2\right)^{[5 \sim 7]}$ 和过氧化脲 $\left[\left(\mathrm{CO}\left(\mathrm{NH}_{2}\right)_{2} \cdot \mathrm{H}_{2} \mathrm{O}_{2}\right.\right.$,
3) $]^{[8]}$ 等.

过氧化脲(urea-hydrogen peroxide, UHP), 又称过氧 化尿素、尿素过氧化氢加合物(urea-hydrogen peroxide adduct 或 urea-hydrogen peroxide complex), 外观为白色 结晶性粉末, 熔点 $84 \sim 86{ }^{\circ} \mathrm{C}$, 制备方法简单 ${ }^{[9]}$. 过氧化 腿可溶于乙醚、乙醇、丙醖、二氯甲烷等有机溶剂中、 $\mathrm{H}_{2} \mathrm{O}_{2}$ 含量高 $(36.2 \%)$ 、常温下稳定性好、活性氧释放可 控等特点 ${ }^{[10]}$, 被认为是高浓度 $\mathrm{H}_{2} \mathrm{O}_{2}$ 溶液和间氯过氧苯 甲酸 ( $m$-chloroperoxybenzoic acid, $m$-CPBA)、过氧三氟乙 酸(trifluoroperacetic acid，TFPPA)等有机过氧酸的理想 替代物 ${ }^{[8]}$. 本文从有机反应类型的角度, 介绍近年来过 氧化脲在各种氧化反应中的应用.

* E-mail: supmoney@163.com

Received March 24, 2011; revised May 3, 2011; accepted October 8, 2011.

Project supported by the Aid Program for Key Science and Technology Innovative Research Team in Zhejiang Province (No. 2009R50002) and the Fundamental Research Funds for the Central Universities of China (No. 2011QNA4018).

浙江省重点科技创新团队(No. 2009R50002)和中央高校基本科研业务费专项资金(No. 2011QNA4018)资助项目. 
<smiles>[NH3+][Pb]1(O)OO[Pb](O)(O)OO1</smiles>

1

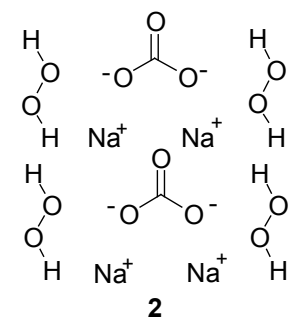

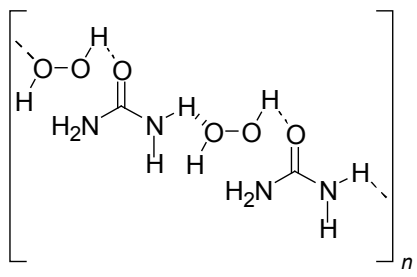

3

\section{1 碳一碳双键环氧化反应}

碳一碳双键的环氧化反应在有机合成中占有重要地 位, 有机环氧化合物结构中的环氧基活性高, 能与多种 物质反应, 可在碳链上一次性引入两个官能团. 该类反 应最常用的氧化剂是过氧化合物, 包括 $\mathrm{H}_{2} \mathrm{O}_{2}$ 、过氧酸和 过氧化醇等 ${ }^{[11]}$. $\mathrm{H}_{2} \mathrm{O}_{2}$ 氧化烯烃的反应常在水中或与水混 溶的溶剂中进行, 但由于 $\mathrm{H}_{2} \mathrm{O}_{2}$ 的浓度较低以致反应较 慢, 常需加入金属催化剂以加速反应 ${ }^{[2]}$. 由于 UHP 可溶 于有机溶剂, 以它为 $\mathrm{H}_{2} \mathrm{O}_{2}$ 源, 反应较易进行, 加入合适 的催化剂亦可提高反应速率, 其中使用最多的是甲基三 氧化铼(methyltrioxorhenium, MTO).

Adam 等 ${ }^{[12]}$ 最早报道了 MTO 催化下, UHP 为氧化剂 的烯烃环氧化反应, 并从该反应的机理(Scheme 1)分析, 认为用 UHP 代替 $\mathrm{H}_{2} \mathrm{O}_{2}$ 溶液可以抑制其中的水解副反应. 环已烯、茨烯、苯乙烯、二苯乙烯等烯烃 $\mathbf{4}$ 的环氧化反 应中, 反应物物质的量比为: 烯烃 : UHP : MTO $=1$ : $1: 0.01$, 在 $\mathrm{CDCl}_{3}$ 中反应 $18 \mathrm{~h}$ 以上, 转化率在 $46 \%$ $98 \%$ 之间, 主产物均为环氧化合物 5 . 若以 $85 \% \mathrm{H}_{2} \mathrm{O}_{2}$ 水
溶液为氧化剂, 则底物转化率低, 且产物是环氧化合物 的水解产物二醇 6 (表 1$)$.

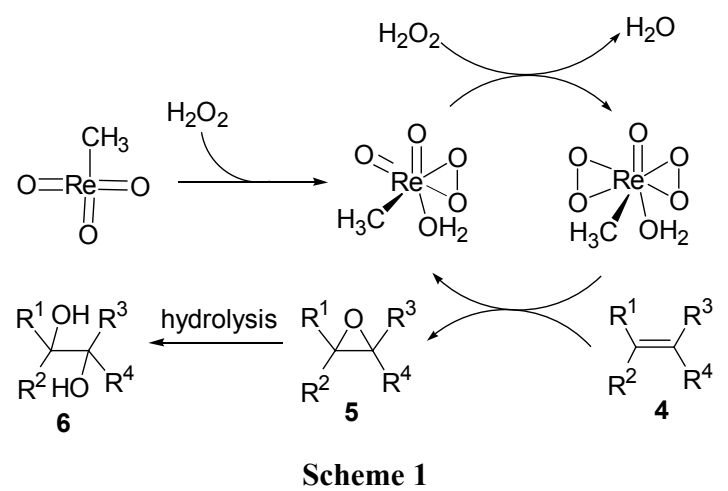

对于在质子溶剂中易分解的物质，可溶于非质子溶 剂的 UHP 可作为理想的氧化剂. Boehlow 等 ${ }^{[13]}$ 报道了将 MTO/UHP 体系用于复杂化合物中双键的环氧化反应. 在对底物胆甾醇(cholesterol, 7)的环氧化中, 发现在二氯 甲烷中底物可以在反应 $1.5 \mathrm{~h}$ 后完全转化, 产物中 $\alpha / \beta=$ $4: 1$; 在甲苯中反应 $3.5 \mathrm{~h}$ 后转化率为 $86 \%$, 产物中 $\alpha / \beta=4: 1$, 这与采用传统氧化剂单过氧邻苯二甲酸镁 (magnesium monoperoxyphalate, MMPP)和二甲基过氧 化酩(dimethyldioxirane, DMD)的结果相近(Eq. 1). 当底 物为愈创木醇 (guaiol, 9)时, 选择性更好, 结果优于传统 的氧化剂 $m$-CPBA 和 MMPP (Eq. 2).

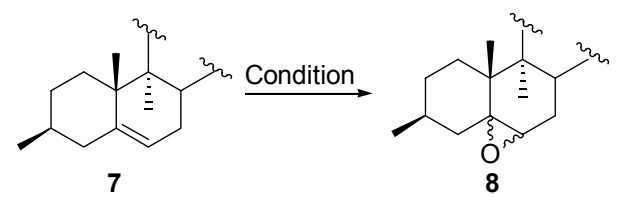

Conditions:

A: UHP (3 equiv.), MTO (5 mol\%), $\mathrm{CH}_{2} \mathrm{Cl}_{2}, 1.5 \mathrm{~h}, \alpha / \beta=4: 1,100 \%$ B: UHP (3 equiv.), MTO (5 mol\%), PhMe, $3.5 \mathrm{~h}, \alpha / \beta=4: 1,86 \%$

C: MMPP $, \alpha / \beta=4: 1,88 \%$

D: DMD, $\alpha / \beta=1: 1,88 \%$

表 1 MTO 催化的烯烃环氧化反应(UHP 为氧化剂)

Table 1 MTO-catalyzed epoxidation of olefins with UHP

\begin{tabular}{|c|c|c|c|c|c|}
\hline Entry & Olefin 4 & Oxidant & Conv. $/ \%$ & Epoxide 5 & Diol 6 \\
\hline 1 & \multirow{2}{*}{ Cyclohexene } & UHP & 98 & 99 & 1 \\
\hline 2 & & $\mathrm{H}_{2} \mathrm{O}_{2}(85 \%)$ & 71 & 0 & 82 \\
\hline 3 & 1-Methylcyclohexene & UHP & 88 & 96 & 4 \\
\hline 4 & \multirow{2}{*}{ Camphene } & UHP & 92 & $\geqslant 95$ & 0 \\
\hline 5 & & $\mathrm{H}_{2} \mathrm{O}_{2}(85 \%)$ & 27 & 0 & 0 \\
\hline 6 & Styrene & UHP & 46 & $\geqslant 95$ & 0 \\
\hline 7 & Indene & UHP & 51 & $\geqslant 95$ & 0 \\
\hline 8 & 1,1-Diphenylethene & UHP & 81 & 93 & 7 \\
\hline 9 & trans-Stilbene & UHP & 44 & $\geqslant 95$ & 0 \\
\hline 10 & trans-Cinnamyl alcohol & UHP & 66 & $\geqslant 95$ & 0 \\
\hline
\end{tabular}




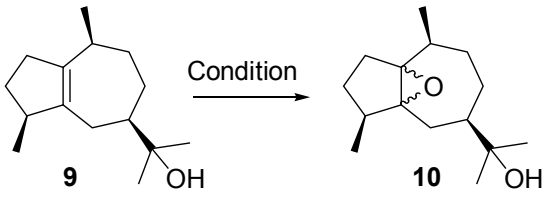

Conditions:

A: UHP (3 equiv.), MTO (5 mol\%), $\mathrm{CH}_{2} \mathrm{Cl}_{2}, 1.5 \mathrm{~h}, \alpha / \beta=9: 1,96 \%$

B: $m$-CPBA, $\alpha / \beta=6: 4$, high yield

C: MMPP, $\alpha l \beta=69: 31,24 \%$

富勒烯 $\left(\mathrm{C}_{60}, 17\right)$ 的环氧化反应是对其进行结构衍生 的重要方法, 一般采用 $\mathrm{O}_{3}{ }^{[14]}, m-\mathrm{CPBA}^{[15]}$ 等氧化剂, 收 率都在较低的水平, 条件也较为苛刻. Murray 等 ${ }^{[16]}$ 将 $\mathrm{MTO} / \mathrm{UHP}$ 体系应用于该反应(Eq. 3), 使 $\mathrm{C}_{60}$ 在极为温和 的条件下转化成 $\mathrm{C}_{60} \mathrm{O}(\mathbf{1 8})$, 最佳的 $\mathrm{C}_{60} \mathrm{O}$ 收率为 $35.4 \%$.

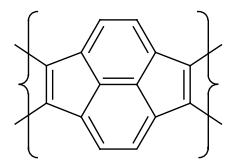

17

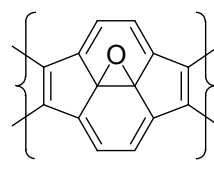

18
除了 $\mathrm{MTO}$, 一些含氟醇类可以通过氢键活化 $\mathrm{H}_{2} \mathrm{O}_{2}$, 包括 UHP 晶体中的 $\mathrm{H}_{2} \mathrm{O}_{2}$. Legros 等 ${ }^{[17]}$ 报道了一种环氧 化的高效体系: 过氧化脲/六氟异丙醇(UHP/HFIP). 以 环辛烯为底物, UHP 为氧化剂, HFIP 为溶剂, 反应 $10 \mathrm{~h}$ 后底物完全转化, 若以 $30 \% \mathrm{H}_{2} \mathrm{O}_{2}$ 为氧化剂, 反应 $24 \mathrm{~h}$ 后, 转化率只有 $79 \%$. 当底物为荁烯 (limonene) 和 trans-4-癸烯时结果也令人满意(前者 $7 \mathrm{~h}$ 转化 $100 \%$, 后 者 $48 \mathrm{~h}$ 转化 $90 \%$ ). 当底物为不活泼的端位烯烃时, 需要 加入 $\mathrm{CF}_{3} \mathrm{COC}_{8} \mathrm{~F}_{17}$ 作为催化剂, 可获得 $89 \%$ 以上的收率. 实验结果列于表 2 中.

表 $2 \mathrm{CF}_{3} \mathrm{COC}_{8} \mathrm{~F}_{17}$ 催化的六氟异丙醇中的烯烃环氧化反应 (UHP 为氧化剂)

Table 2 Catalyzed epoxidation reaction with $\mathrm{CF}_{3} \mathrm{COC}_{8} \mathrm{~F}_{17}$ and UHP in HFIP

\begin{tabular}{clcccc}
\hline Entry & \multicolumn{1}{c}{ Olefin 4 } & Cat./mol\% & Temp. $/{ }^{\circ} \mathrm{C}$ & Time $/ \mathrm{h}$ & Yield $\%$ \\
\hline 1 & & 0 & 25 & 7 & 88 \\
2 & Limonene & 3 & 25 & 0.75 & 79 \\
3 & Cyclooctene & 3 & 25 & 1 & 85 \\
4 & & & & & \\
5 & trans-4-Decylene & 3 & 25 & 48 & 90 \\
& & & 25 & 5 & 93 \\
6 & Ethyl undec-10- & 0 & 25 & 48 & - \\
7 & enoate & 5 & 40 & 24 & 81 \\
8 & Dodec-1-ene & 5 & 40 & 24 & 94 \\
9 & Allylbenzene & 5 & 40 & 48 & 92 \\
\hline
\end{tabular}

UHP 和各种酸酐组成的体系也常用于环氧化反应, 其中的活性物种是 UHP 与酸酐原位生成的过氧酸. 2003 年 Fan 等 ${ }^{[18]}$ 报道了 $N$-methacryloylpyrazolidinone 衍生物
19 的不对称环氧化反应, 有 UHP 参与的氧化体系 $\mathrm{UHP} / \mathrm{TFAA}, \mathrm{UHP} / \mathrm{TFSA}, \mathrm{UHP} /\left(\mathrm{CCl}_{3} \mathrm{CO}\right)_{2} \mathrm{O}$ 均有较好的 反应效果, 优于 $m$-CPBA 和 dioxirane 等氧化剂(Eq. 4).<smiles>[R]C([R])=C([R])C(=O)N1C2CCC(C)(C)CC(C2)C1([R])C</smiles>

19<smiles>[R]C12CCC(C)(CN(c3ccccc3)C1=O)CN2C(=O)C1([R])OC1([R])[R]</smiles>

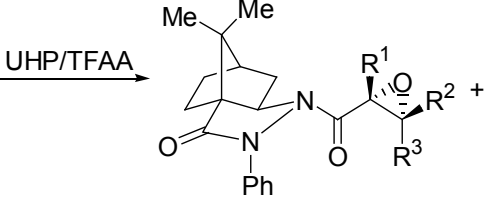

20

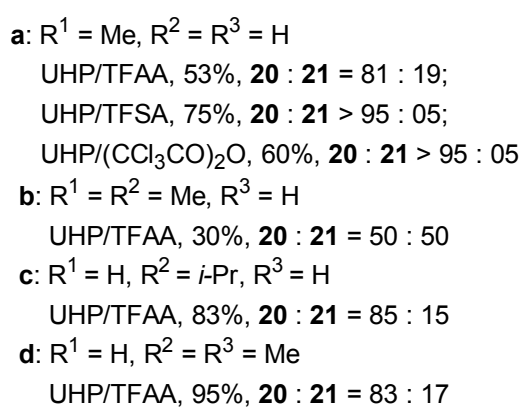

2003 年 Valderrama 等 ${ }^{[19]}$ 报道了 UHP 用于醌类化合 物的环氧化反应(Eq. 5), 以 $\mathrm{UHP} / \mathrm{K}_{2} \mathrm{CO}_{3}$ 为氧化试剂, 甲 醇为溶剂, 室温下反应, 所得到的收率均在 $50 \%$ 以上.<smiles>[R]C1=CC(=O)c2c([R2])ccc([R2])c2C1=O</smiles>

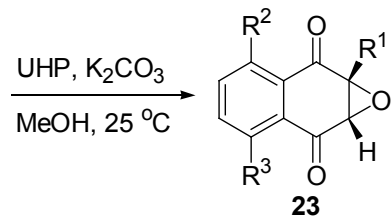

2006 年 Ankudey 等 ${ }^{[20]}$ 报道了脂肪酶 Novozyme 435 催化的以 UHP 为氧化剂的环氧化反应(Scheme 2), 收率 在 $73 \% \sim 100 \%$ 之间. 酶催化的反应时间一般较长，反应 所需 $\mathrm{H}_{2} \mathrm{O}_{2}$ 可由 UHP 缓慢释放. 同时无水体系抑制了环 氧化产物的水解反应，提高了收率.

2009 年 Jin 等 ${ }^{[21]}$ 研究了在超声条件下, 使用 UHP 对查尔酮(24)及其衍生物进行环氧化反应的方法(Eq. 6). 以乙醇一水混合物为溶剂，对各种查尔酮类底物进行环 氧化，收率在 $78 \%$ ～93\%之间，超声能明显提升反应效 果(括号中的收率代表未加超声条件).

2010 年, Mahmoodi 等 ${ }^{[22]}$ 报道了使用 UHP 对 1,4-二 


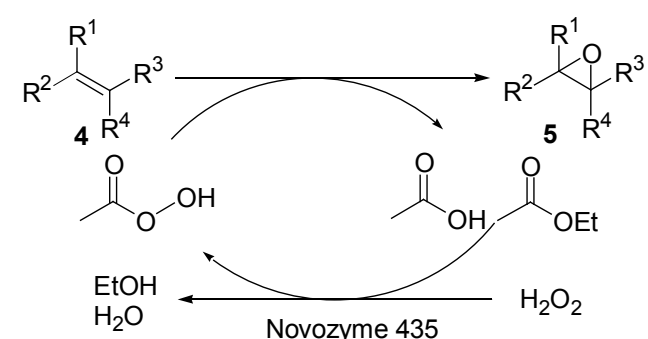

Scheme 2

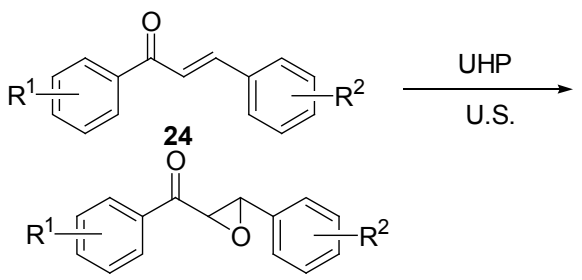

Without ultrasound the yields indicated in parenthesis

$$
\begin{aligned}
\mathrm{R}^{1} & =\mathrm{H}, \mathrm{R}^{2}=\mathrm{H}, 91 \%(83 \%) ; \\
\mathrm{R}^{1} & =4-\mathrm{F}, \mathrm{R}^{2}=\mathrm{H}, 87 \%(78 \%) ; \\
\mathrm{R}^{1} & =4-\mathrm{F}, \mathrm{R}^{2}=3-\mathrm{NO}_{2}, 86 \%(71 \%) ; \\
\mathrm{R}^{1} & =4-\mathrm{F}, \mathrm{R}^{2}=2,4-\mathrm{Cl}_{2}, 89 \%(73 \%) ; \\
\mathrm{R}^{1} & =4-\mathrm{Cl}, \mathrm{R}^{2}=\mathrm{H}, 90 \%(60 \%) ; \\
\mathrm{R}^{1} & =4-\mathrm{Br}, \mathrm{R}^{2}=\mathrm{H}, 85 \%(70 \%) \\
\mathrm{R}^{1} & =4-\mathrm{MeO}, \mathrm{R}^{2}=\mathrm{H}, 93 \%(65 \%) ; \\
\mathrm{R}^{1} & =4-\mathrm{MeO}, \mathrm{R}^{2}=3-\mathrm{NO}_{2}, 84 \%(70 \%) \\
\mathrm{R}^{1} & =2,4-\mathrm{Cl}_{2}, \mathrm{R}^{2}=4-\mathrm{MeO}, 78 \%(45 \%) ; \\
\mathrm{R}^{1} & =2,4-\mathrm{Cl}_{2}, \mathrm{R}^{2}=3-\mathrm{NO}_{2}, 85 \%(75 \%)
\end{aligned}
$$

芳酰基乙烯(26)进行环氧化的方法(Eq. 7). 反应条件类 似于 Valderrama 等 ${ }^{[19]}$ 在醌类化合物环氧化中使用的方 法, 反应在乙醇中室温下进行, 加入 $\mathrm{NaOH}$ 作为催化剂, 产物 27 收率处于中等水平 $(55 \% \sim 76 \%)$.

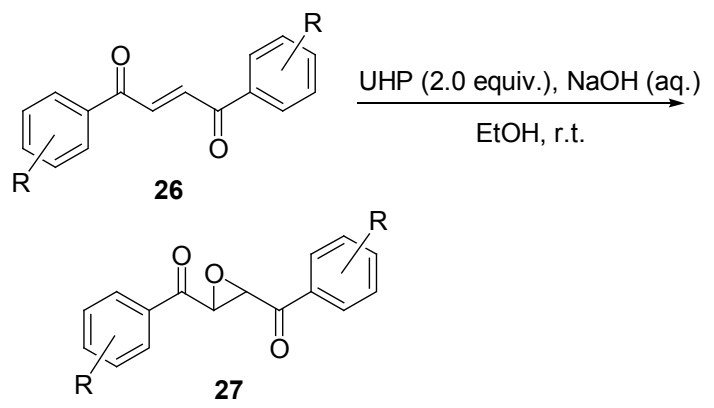

$\mathrm{R}=\mathrm{H}, 6 \mathrm{~h}, 76 \% ; \mathrm{R}=2,4-\mathrm{Me}_{2}, 14 \mathrm{~h}, 75 \%$; $\mathrm{R}=4-\mathrm{Cl}, 15 \mathrm{~h}, 60 \% ; \mathrm{R}=4-\mathrm{Br}, 20 \mathrm{~h}, 55 \%$

烯烃的环氧化反应是最早应用 UHP 的领域之一, 在反应条件选择、底物影响等方面均已相对成熟. 目前 该类研究除了上述的反应外, 主要还集中于催化剂的改 进与开发上, 如催化剂的负载 ${ }^{[23 ~ 25]}$ 、加入氮杂环作为碱

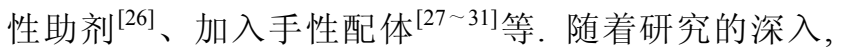
UHP 在烯烃环氧化反应中的应用将得到不断的拓展.

\section{Baeyer-Villiger 氧化反应}

在 Baeyer-Villiger 氧化反应(简称 B-V 反应)中, 最 常用的氧化剂是 $90 \%$ 的 TFPPA 和 $85 \%$ 的 $m$-CPBA 等有 机过氧酸. UHP 有较高的 $\mathrm{H}_{2} \mathrm{O}_{2}$ 含量, 且属于无水体系, 用于 $\mathrm{B}-\mathrm{V}$ 反应可以避免高浓度 $\mathrm{H}_{2} \mathrm{O}_{2}$ 带来的危险性，也 可使反应在无水条件下进行, 是上述氧化剂的理想替代 物, 且以 UHP 为活性氧源的 B-V 反应已在众多有机化 合物的合成中得到了应用.

环酮的 B-V 反应是合成环内酯的一种有效方法, 由 于羰基两侧碳原子的绝对构型在反应前后保持不变, 因 此环酮的立体选择性 B-V 反应具有重要的合成价值. 2004 年 Marigo 等 ${ }^{[32]}$ 报道了 4,5-二苯基咪唑啉(4,5-DPI) 催化下酮的对映体选择性 $\alpha$-氯化反应，在得到产物 $(R)-\alpha$-氯代环己酩(28)后，进行一系列的衍生化反应，其 中采用 UHP/TFAA 体系进行 B-V 反应，以 $81 \%$ 的收率 立体选择性地得到产物氯代内酯 29 (Eq. 8).

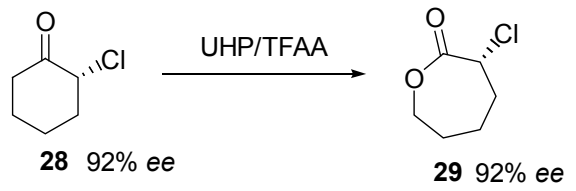

2007 年 Rios 等 ${ }^{[33]}$ 报道了固载化的脂肪酶 Novozyme-435 催化的环己酮 B-V 反应(Eq. 9), 结果表 明, 以 UHP 为氧化剂, 乙酸乙酯为溶剂, 不仅在烯烃环 氧化反应中效果较好 ${ }^{[20]}$, 在环已酮及取代环已酮 30 的 B-V 反应也具有很高的催化效率. 反应介质的无水条件 避免了氧化产物的水解. 在该反应的催化循环中, UHP 在脂肪酶 Novozyme-435 的催化下将乙酸乙酯(或催化循 环中得到的乙酸)转化成过氧乙酸, 后者将环己酮经 $\mathrm{B}-\mathrm{V}$ 反应转化成环内酯, 所得的乙酸继续进入催化循 环.

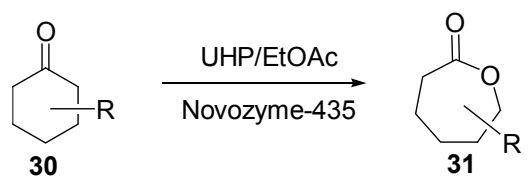$$
31
$$

$\mathrm{R}=6-\mathrm{H}, 80 \% ; \mathrm{R}=4-\mathrm{Ph}, 75 \% ; \mathrm{R}=\mathrm{Me}, 95 \%$;

$\mathrm{R}=6$-sec-Bu, 78\%; R = 6-tert-Bu, 8\%; R = 6-Allyl, 92\%;

$R=6-B n, 93 \% ; R=6-P h, 94 \% ; R=6,6-M_{2}, 96 \%$

由于甲基不易迁移，甲基酮的 B-V 反应作为在碳链 上减少两节碳的有效方法，而甲基酮比较容易获得，因 此该方法在有机合成中得到广泛应用, UHP/TFAA 常用 于这类反应. Marcos 等 ${ }^{[34]}$ 报道了从核盘菌素(sclareol)高 效合成(+)-nimbiol 及其他罗汉松烷(podocarpane)衍生 物的方法, 从乙酰化衍生物 32 得到乙酸酯 33 的过程中, 采用了 UHP/TFAA 体系, 在室温下反应 $24 \mathrm{~h}$, 以 $91 \%$ 的 收率得到产物 33 (Eq. 10). 


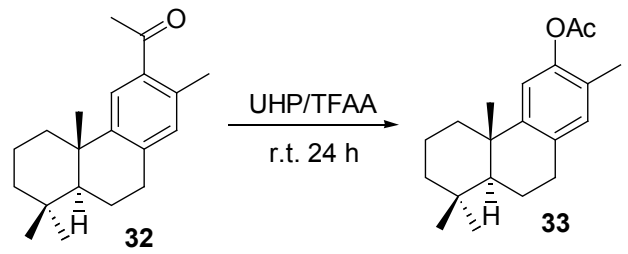

Garrido 等 ${ }^{[35]}$ 报道了一种立体选择性合成生物活性 物质(+)-L-733060 和(+)-CP-99994 的方法, 在以甲基 酮 34 合成乙酸酯 35 的反应, 采用了 UHP/TFAA 体系, 以 $\mathrm{CH}_{2} \mathrm{Cl}_{2}$ 为溶剂, $\mathrm{Na}_{2} \mathrm{HPO}_{4}$ 为缓冲剂, 以 $60 \%$ 的收率合 成了目标产物(Eq. 11).

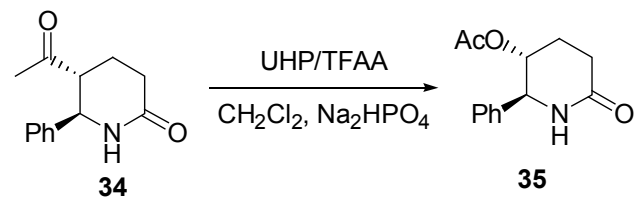

B-V 反应也是较早应用 UHP 的反应, 在全合成中 的较多实例某种程度上证明了 UHP 所参与反应结果的 稳定性. 但 UHP 与酸酕组成的体系中, 后者是化学计量 的, 因此诸如上述脂肪酶等能够活化 UHP 的催化剂将 是研究的重点.

\section{3 硫醚氧化反应}

亚砜与砜的主要合成方法是氧化相应的硫醚, 两者 的选择性与所选用的氧化剂和催化剂密切相关. 用等量 的 $30 \% \mathrm{H}_{2} \mathrm{O}_{2}$ 或其他氧化试剂, 如 $\mathrm{NaIO}_{4}, \mathrm{NaBO}_{3} \cdot 4 \mathrm{H}_{2} \mathrm{O}$, $m$-CPBA 等均可将硫醚氧化成亚砜. 再用 1 倍量的 $\mathrm{H}_{2} \mathrm{O}_{2}$, $\mathrm{NaBO}_{3} \cdot 4 \mathrm{H}_{2} \mathrm{O}, \mathrm{KMnO}_{4}$ 等氧化剂可以将亚砜继续氧化成 砜. 如果有足够量的氧化剂(通常在 3 倍以上), 可以不 分离亚砜将硫醚直接氧化成砜.

1998 年 Gunaratne 等 ${ }^{[36]}$ 报道了采用 UHP/MTO 体系 在室温下将多种硫醚 36 氧化成相应的亚砜 37 , 收率在 $80 \% \sim 92 \%$ 之间(表 3). 该体系对酯基有很好的相容性， 对于含双键的硫醚并无环氧化副产物的产生, 手性底物 在手性中心保持原有构型的前提下也能有较好的收率.

1999 年 Balicki ${ }^{[37]}$ 报道了使用 UHP/85\% $\mathrm{HCOOH}$ 体 系在室温下将硫醚 $\mathbf{3 6}$ 氧化成相应的砜 $\mathbf{3 8}$, 产物中未检 测到亚砜, 且若将 UHP 换成 $30 \% \mathrm{H}_{2} \mathrm{O}_{2}$, 反应的收率显 著降低, 甚至不发生反应. 在此基础上, Balicki ${ }^{[38]}$ 研究 了在乙腈中以 UHP/TFAA 为氧化剂将硫醚氧化成砜的 反应，反应时间 $0.5 \sim 4 \mathrm{~h}$, 收率在 $90 \% \sim 98 \%$ 之间. 为了 便于比较, 两种方法列于表 4 中.

1999 年 Varma 等 ${ }^{[39]}$ 实现了在无溶剂条件下, UHP 对硫醚 36 的选择性氧化, UHP 与硫醚的比例为 $2: 1$, 在 $85{ }^{\circ} \mathrm{C}$ 下短时间反应可得到产物亚砜 37, 收率在 $80 \%$ $87 \%$ 之间, 主要的副产物为 $10 \%$ 左右的砜 38 ; 同样的
表 3 MTO 催化硫醚氧化成亚砜的反应(UHP 为氧化剂) Table 3 Oxidation of sulfides to sulfoxides with UHP catalyzed by MTO

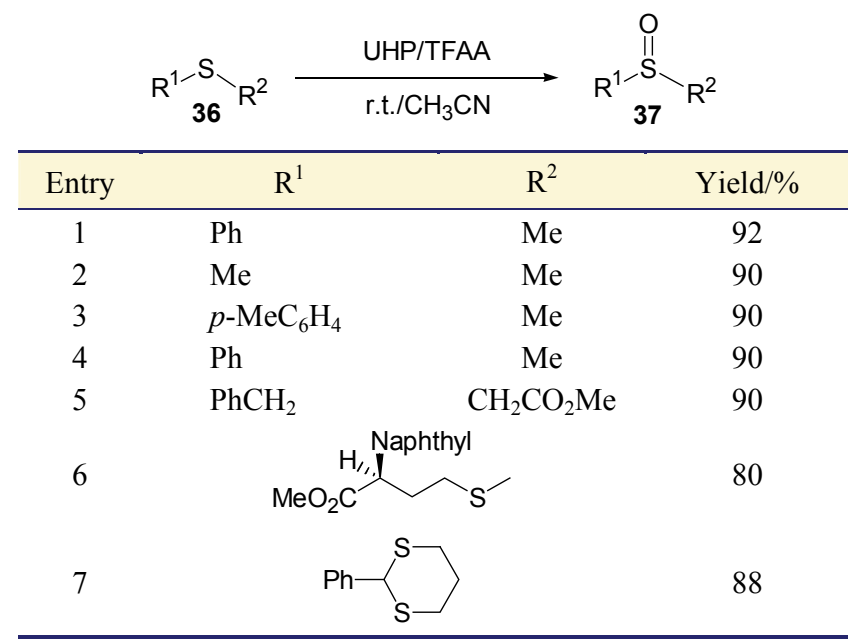

表 $4 \mathrm{UHP} / \mathrm{HCOOH}$ 和 UHP/TFAA 体系氧化硫醚成砜的反应 Table 4 Oxidation of sulfides to sulfones with UHP/HCOOH and UHP/TFAA

\begin{tabular}{|c|c|c|c|c|}
\hline \multirow{2}{*}{ Entry } & \multirow{2}{*}{$\mathrm{R}^{1}$} & \multirow{2}{*}{$\mathrm{R}^{2}$} & \multicolumn{2}{|c|}{ Yield/\% } \\
\hline & & & $\mathrm{A}^{a}$ & $\mathrm{~B}^{b}$ \\
\hline 1 & $n-\operatorname{Pr}$ & $n-\operatorname{Pr}$ & 96 & 98 \\
\hline 2 & $n$-Bu & $n$-Bu & 95 & 95 \\
\hline 3 & $t-\mathrm{Bu}$ & $t-\mathrm{Bu}$ & 92 & 96 \\
\hline 4 & $i-\operatorname{Pr}$ & $i-\operatorname{Pr}$ & 94 & 93 \\
\hline 5 & $\mathrm{Me}$ & $\mathrm{Ph}$ & 95 & - \\
\hline 6 & $\mathrm{Me}$ & $\operatorname{Pr}$ & - & 97 \\
\hline 7 & $\mathrm{Me}$ & $\mathrm{Bn}$ & - & 96 \\
\hline 8 & $\mathrm{Bn}$ & $\mathrm{Bn}$ & 97 & 98 \\
\hline 9 & $\mathrm{Bn}$ & $\mathrm{Ph}$ & 94 & 95 \\
\hline 10 & $\mathrm{Ph}$ & $\mathrm{Ph}$ & 95 & 98 \\
\hline 11 & $o-\mathrm{NO}_{2} \mathrm{C}_{6} \mathrm{H}_{4}$ & $\mathrm{Ph}$ & 90 & 92 \\
\hline 12 & $p-\mathrm{NO}_{2} \mathrm{C}_{6} \mathrm{H}_{4}$ & $p-\mathrm{NO}_{2} \mathrm{C}_{6} \mathrm{H}_{4}$ & 91 & 91 \\
\hline 13 & & & 98 & 98 \\
\hline 14 & & & 88 & 90 \\
\hline 15 & & & 96 & 97 \\
\hline
\end{tabular}

${ }^{a} \mathrm{~A}=\mathrm{UHP} / 85 \% \mathrm{HCOOH}^{[37]} ;{ }^{b} \mathrm{~B}=\mathrm{UHP} / \mathrm{TFAA}^{[38]}$.

温度下反应较长时间可得到产物砜, 收率在 $87 \%$ 90\% 之间(Scheme 3).

2004 年 Sasaki 等 ${ }^{[00]}$ 报道了以负载于氟磷灰石(FAp) 上的磷钿酸四丁基铵为催化剂, 利用 UHP 将硫醚 $\mathbf{3 6}$ 无 溶剂氧化成亚砜 37 和砜 38 的反应(Scheme 4). 该体系 


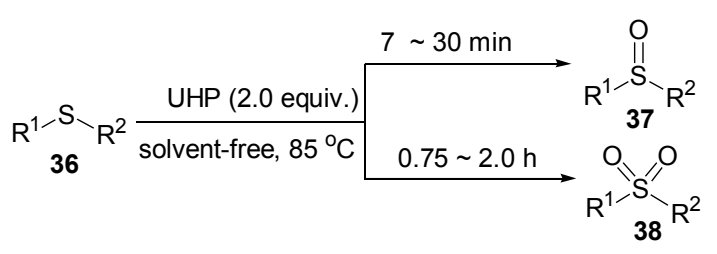

Scheme 3

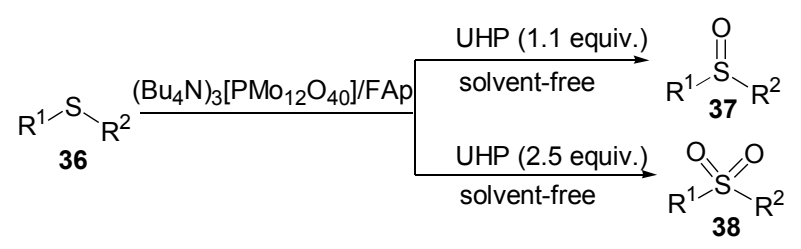

Scheme 4

可以在无溶剂、中性的温和条件下完成硫醚的选择性氧 化, 用 UHP 的加入量控制产物的氧化程度, 反应后处理 简单，亚砜的收率在 $49 \% \sim 93 \%$ 之间，砜的收率在 $62 \% \sim 99 \%$ 之间.

最近, Hasaninejad 等 ${ }^{[41}$ 报道了以硫酸氢䐂盐(UHS) 为催化剂, 利用 UHP 将硫梄氧化成亚砜的反应(Eq. 12). 实验结果表明, UHP/UHS 体系可以在甲醇中室温下以 极高的选择性完成硫醚氧化成亚砜的反应, 收率在 $80 \% \sim 95 \%$ 之间，且对醛基等易氧化官能团有很好的相 容性.

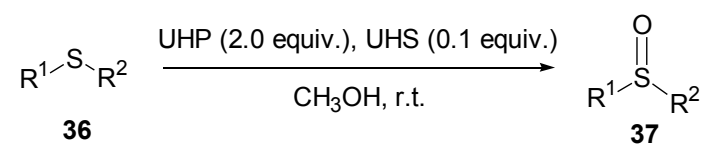

在上述以硫醚为原料制备亚砜的反应中, 若在氧化 剂中加入手性添加剂, 则可以生成非外消旋的亚砜. 若 在催化剂中加入手性配体, 可实现从硫醚制备光学活性 的亚砜 ${ }^{[42,43]}$, 该类不对称氧化反应将有较大的研究意 义.

\section{4 氮原子的氧化反应}

\section{1 含氮杂环的反应}

含氮杂环化合物经 $N$-氧化反应生成的 $N$-氧化物具 有独特的性能, 在含能材料、有机合成和高分子化学等 领域都有广泛的应用 ${ }^{[44]}$. 现有制备 $N$-氧化物的方法较 多, 但存在反应不完全、产物收率和纯度低、氧化试剂 价格昂贵且易爆等问题.

1992 年 Kaczmarek 等 ${ }^{[45]}$ 报道了用过氧化脲/邻苯二 甲酸䣶(UHP/phthalic anhydride)体系氧化氮杂芳香环和 叔胺的反应. 在 UHP 用量为底物的 $3.3 \sim 4.5$ 倍, 酸䣶用 量为底物的 $1.1 \sim 1.5$ 倍条件下, 获得了很好的结果. 作 者还尝试了乙酸䣶、苯甲酸䣶和马来酸酕等其他有机酸
酐，反应效果均比不上邻苯二甲酸酐. 若用 $30 \% \mathrm{H}_{2} \mathrm{O}_{2}$ 替换 UHP，反应无法进行.

1999 年 Varma 等 ${ }^{[39]}$ 报道了用 UHP 在无溶剂条件下 氧化吡啶生成 $N$-氧化吡啶的反应, UHP 用量为 2 倍, 在 $85{ }^{\circ} \mathrm{C}$ 下反应 $45 \mathrm{~min}$, 以 $87 \%$ 的收率得到氧化产物, 同 样的条件应用于四甲基吡嗪的 $N$-氧化反应, 可以获得 $92 \%$ 的收率. 但该反应未作进一步的拓展.

适用于富电子吡啶环进行 $N$-氧化反应的体系在应 用于缺电子吡啶环时, 往往反应效果较差, 人们把研究 的突破口放在了 UHP 与酸酲的组合上. 2000 年 Caron 等 ${ }^{[46]}$ 报道了用 UHP/TFAA 体系氧化缺电子吡啶环生成 相应的 $N$-氧化吡啶的反应. 经过实验发现, 该体系在氧 化缺电子吡啶环反应中明显优于 $\mathrm{MTO} / 30 \% \mathrm{H}_{2} \mathrm{O}_{2}$, Oxone, UHP/TFA, UHP/Ac $/ \mathrm{Ac}_{2} \mathrm{O}, 30 \% \mathrm{H}_{2} \mathrm{O}_{2} / \mathrm{TFAA}, 70 \%$ $t$-BuOOH 等氧化试剂.

2000 年 Balicki 等 ${ }^{[47]}$ 报道了用 UHP $/ 95 \% \mathrm{HCOOH}$ 体 系氧化氮杂芳环的反应. 该体系不仅能将吡啶环氧化成 相应的 $N$-氧化吡啶，也能将二溙类化合物氧化成相应 的单氧化产物. 实验中发现若将 UHP 换成 $30 \% \mathrm{H}_{2} \mathrm{O}_{2}$, 反应效果显著下降甚至不发生反应.

2008 年 Rong 等 ${ }^{[48]}$ 报道了 UHP/TFAA 体系氧化吡 啶生成 $N$-氧化吡啶的反应, 作者研究了溶剂对反应的 影响, 发现底物在氯仿和二氧六环中均可完全转化, 收 率也有较高水平.

上述研究工作中由吡啶衍生物 39 制备 $N$-氧化吡啶 40 的结果列于表 5 中, 以便比较.

表 5 UHP 氧化吡定成相应 $N$-氧化吡啶的反应

Table 5 Oxidation of pyridines to their $N$-oxides with UHP

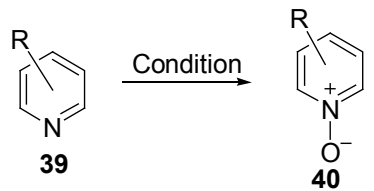

Conditions: A: UHP (1.1 equiv.), phthalic anhydride (1.1 equiv.), $\mathrm{CH}_{2} \mathrm{Cl}_{2}$ or $\mathrm{CH}_{3} \mathrm{CN}$, r.t.; B: UHP (2.1 equiv.), TFAA (2.0 equiv.), $\mathrm{CH}_{2} \mathrm{Cl}_{2}$ or $\mathrm{CH}_{3} \mathrm{CN}, 0^{\circ} \mathrm{C}$; C: UHP (7.0 equiv.), $95 \% \mathrm{HCOOH}$, r.t.; D: UHP (10.0 equiv.), $\mathrm{K}_{2} \mathrm{CO}_{3}$ (20.0 equiv.),1,4-dioxane or $\mathrm{CHCl}_{3}$, $50{ }^{\circ} \mathrm{C}$

\begin{tabular}{ccccccc}
\hline & & \multicolumn{5}{c}{ Yield/\% } \\
\cline { 3 - 7 } Entry & $\mathrm{R}$ & $\mathrm{A}^{[45]}$ & $\mathrm{B}^{[46]}$ & $\mathrm{C}^{[47]}$ & \multicolumn{2}{c}{$\mathrm{D}^{[48]}$} \\
\hline 1 & $3-\mathrm{Br}$ & - & - & - & 90 & 97 \\
2 & $2-\mathrm{Br}$ & - & - & - & 93 & 97 \\
3 & $2-\mathrm{Cl}$ & 92 & - & - & - & - \\
4 & $4-\mathrm{Cl}$ & - & - & 90 & - & - \\
5 & $2-\mathrm{Me}$ & 95 & - & - & 96 & 96 \\
6 & $3-\mathrm{Me}$ & - & - & - & 94 & 95 \\
7 & $4-t-\mathrm{Bu}$ & - & - & 87 & - & - \\
\hline
\end{tabular}




\begin{tabular}{|c|c|c|c|c|c|c|}
\hline \multirow{3}{*}{ Entry } & \multirow{3}{*}{$\mathrm{R}$} & \multicolumn{5}{|c|}{ Yield $/ \%$} \\
\hline & & \multirow{2}{*}{$\mathrm{A}^{[45]}$} & \multirow{2}{*}{$\mathrm{B}^{[46]}$} & \multirow{2}{*}{$C^{[47]}$} & \multicolumn{2}{|c|}{$\mathrm{D}^{[48]}$} \\
\hline & & & & & $\mathrm{CHCl}_{3}$ & Dioxane \\
\hline 8 & 4-Ph & - & - & 77 & - & - \\
\hline 9 & $2-\mathrm{NO}_{2}$ & - & - & 74 & - & - \\
\hline 10 & $2-\mathrm{MeO}$ & 48 & - & - & - & - \\
\hline 11 & $3-\mathrm{MeO}$ & - & - & 82 & - & - \\
\hline 12 & $2,6-\mathrm{Me}_{2}$ & - & - & - & 96 & 95 \\
\hline 13 & $3,5-\mathrm{Me}_{2}$ & - & - & 85 & - & - \\
\hline 14 & $3,5-\mathrm{Br}_{2}$ & 96 & - & - & - & - \\
\hline 15 & $3-\mathrm{CO}_{2} \mathrm{Et}, 5-\mathrm{Br}$ & - & 91 & - & - & - \\
\hline 16 & $2-\mathrm{Cl}, 3-\mathrm{CN}$ & - & 70 & - & - & - \\
\hline 17 & $2-\mathrm{Br}, 5-\mathrm{NO}_{2}$ & - & 82 & - & - & - \\
\hline 18 & 2-MeO, 5- $\mathrm{NO}_{2}$ & - & 56 & - & - & - \\
\hline 19 & $2-\mathrm{Cl}, 5-\mathrm{CF}_{3}$ & - & 82 & - & - & 一 \\
\hline 20 & $2,6-\left(\mathrm{CO}_{2} \mathrm{Me}\right)_{2}$ & - & 55 & - & - & - \\
\hline 21 & $2,4-\left(\mathrm{CO}_{2} \mathrm{Me}\right)_{2}$ & - & 98 & - & - & - \\
\hline 22 & $3,4-\left(\mathrm{CO}_{2} \mathrm{Et}\right)_{2}$ & - & 98 & - & - & - \\
\hline 23 & $2-\mathrm{Me}, 5-\mathrm{CO}_{2} \mathrm{Me}$ & - & 81 & - & - & 一 \\
\hline 24 & $4-\mathrm{CN}$ & 21 & - & 42 & - & - \\
\hline 25 & $4-\mathrm{CO}_{2} \mathrm{Et}$ & 90 & 83 & 85 & - & - \\
\hline 26 & $3-\mathrm{CO}_{2} \mathrm{Me}$ & 98 & - & 88 & - & - \\
\hline 27 & $3-\mathrm{CONH}_{2}$ & 80 & - & 79 & - & - \\
\hline 28 & $3-\mathrm{CONEt}_{2}$ & - & 63 & - & - & - \\
\hline 29 & 3-COMe & - & 48 & 68 & - & - \\
\hline 30 & 4-COMe & 95 & - & - & - & - \\
\hline 31 & 3-COPh & - & 98 & - & - & - \\
\hline
\end{tabular}

\section{2 仲胺氧化生成硝酮的反应}

硝酩(nitrone, 41) 是亚胺的 $N$-氧化物, 可作为重要 的 1,3-偶极试剂与烯烃发生 1,3-偶极环加成反应生成异 啞唑啉类杂环化合物. 制备硝酮的常用方法有 $N, N$-二取 代差弪胺的氧化、仲胺的直接氧化、肜与卤代烃反应以及 烷基着胺与羰基化合物反应等.

1995 年 Marcantoni 等 ${ }^{[49}$ 报道了在金属催化下用 UHP 将仲胺 42 氧化成硝酮 41 的反应. 作者选用 $\mathrm{Na}_{2} \mathrm{WO}_{4}, \mathrm{Na}_{2} \mathrm{MoO}_{4}, \mathrm{SeO}_{2}$ 等作为催化剂, 环状和开链的 仲胺均能以较高的收率转化成硝䣶. 该氧化体系对酸性 敏感的保护基团有很好的相容性, 对手性底物也可保持 构型. 1996 年 Goti 等 ${ }^{[50]}$ 报道了在甲基三氧化锞(MTO) 催化下用 UHP 将仲胺 42 氧化成硝䣶 41 的反应. 1996 年 Murray 等 ${ }^{[51]}$ 报道了甲基三氧化锞(MTO)催化下分别 用 $\mathrm{H}_{2} \mathrm{O}_{2}$ 溶液和 UHP 将仲胺 $\mathbf{4 2}$ 氧化成硝酮 41 的反应. 这 三种不同条件下的反应结果详见表 6 .

2007 年 Soldaini 等 ${ }^{[52]}$ 报道了在 MTO 的催化下, 以 UHP 为氧化剂, 选择性地将亚胺 42 氧化成硝酮 41 的反 应. 2008 年 Cardona 等 ${ }^{[53]}$ 报道了在 MTO 的催化下, 以 UHP 为氧化剂, 伯胺 $\mathbf{4 4}$ 与醛 $\mathbf{4 5}$ 一锅反应得到相应的硝 酮化合物. 2009 年 Singh 等 ${ }^{[54]}$ 报道了将氯氧化钿负载于 Nafion 上, 同样以 UHP 为氧化剂, 催化伯胺 44 与醛 45
表 6 金属催化 UHP 氧化仲胺成硝酮的反应

Table 6 Oxidation of secondary amines to nitrones with UHP-metal catalysts

$$
\begin{aligned}
& \text { Conditions: } \\
& \text { A: UHP (4.0 equiv.), metal cat. (0.5 equiv.), } \\
& \text { metal cat.: } \mathrm{W}=\mathrm{Na}_{2} \mathrm{WO}_{4} ; \mathrm{Mo}=\mathrm{Na}_{2} \mathrm{MoO}_{4} ; \mathrm{Se}=\mathrm{SeO}_{2} \\
& \text { B: UHP ( } 3.0 \text { equiv.), MTO (0.02 equiv.), } \mathrm{CH}_{3} \mathrm{OH} \text {, r.t. } \\
& \text { C: UHP (10.0 equiv.), MTO ( } 0.03 \text { equiv.), } \mathrm{CH}_{2} \mathrm{Cl}_{2} \text {, r.t. }
\end{aligned}
$$

\begin{tabular}{|c|c|c|c|c|}
\hline \multirow{2}{*}{ Entry } & \multirow{2}{*}{ Substrate } & \multicolumn{3}{|c|}{ Yield $/ \%$} \\
\hline & & $\mathbf{A}^{[49]}$ & $\mathbf{B}^{[50]}$ & $\mathbf{C}^{[51]}$ \\
\hline 1 & & $92(\mathrm{~W}), 80(\mathrm{Mo})$ & 60 & 95 \\
\hline 2 & & $70(\mathrm{~W}), 45(\mathrm{Mo})$ & - & - \\
\hline 3 & & $84(\mathrm{~W}), 90(\mathrm{Mo})$ & 95 & 85 \\
\hline 4 & & $80(\mathrm{~W}), 87(\mathrm{Mo})$ & 86 & 90 \\
\hline 5 & & $75(\mathrm{~W}), 60(\mathrm{Mo})$ & - & 50 \\
\hline 6 & & - & 70 & - \\
\hline 7 & & $80(\mathrm{~W}), 76(\mathrm{Mo})$ & 82 & 90 \\
\hline 8 & & $60(\mathrm{~W}), 50(\mathrm{Mo})$ & 65 & 80 \\
\hline 9 & & $\begin{array}{l}70(\mathrm{~W}), 62(\mathrm{Se}), \\
55(\mathrm{Mo})\end{array}$ & - & 80 \\
\hline 10 & & $80(\mathrm{~W}), 78(\mathrm{Mo})$ & 60 & 90 \\
\hline 11 & & - & 91 & - \\
\hline
\end{tabular}

缩合氧化直接生成硝酮的反应. 这三种合成硝酮方法的 反应结果列于表 7 中, 以便比较.

\section{5 氧化卤化反应}

卤化反应是有机合成中一类重要的单元反应，常用 的卤化试剂包括卤素单质(氟气因其活性过高一般不采 用，常用的氟化试剂有 Selectfluor，NSFI，DAST， $\mathrm{Et}_{3} \mathrm{~N}-3 \mathrm{HF}$ 等, 碘则需要其他氧化性物质进行活化), $N$-卤 
表 7 金属催化 UHP 氧化亚胺成硝酮的反应

Table 7 Oxidation of imines to nitrones with UHP-metal catalysts

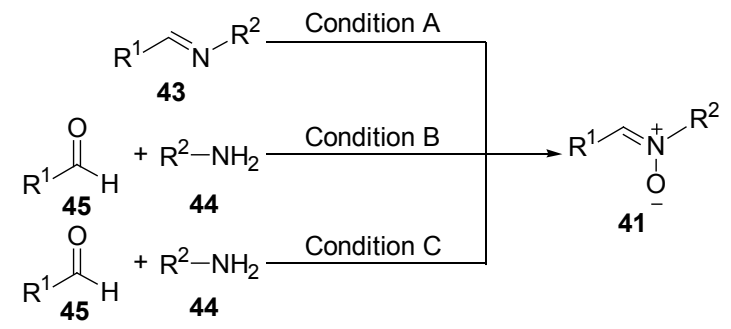

Conditions:

A: UHP (3.0 equiv.), MTO (0.02 equiv.), $\mathrm{MeOH}$

B: amine (1.0 1.2 equiv.), UHP (3.0 equiv.), MTO ( 0.02 equiv.), $\mathrm{CH}_{2} \mathrm{Cl}_{2}$, r.t.

C: amine (1.2 equiv.), UHP (3.0 equiv.), Cat. (0.02 equiv.), $\mathrm{MeOH}$, r.t.

\begin{tabular}{|c|c|c|c|c|c|}
\hline \multirow{2}{*}{ Entry } & \multirow{2}{*}{$\mathrm{R}^{1}$} & \multirow{2}{*}{$\mathrm{R}^{2}$} & \multicolumn{3}{|c|}{ Yield/\% } \\
\hline & & & $\mathrm{A}^{[52]}$ & $\mathrm{B}^{[53]}$ & $\mathrm{C}^{[54]}$ \\
\hline 1 & $\mathrm{Ph}$ & $\mathrm{Bn}$ & 78 & 71 & 80 \\
\hline 2 & $\mathrm{Bn}$ & $n-\mathrm{Bu}$ & - & 80 & - \\
\hline 3 & $\mathrm{Ph}$ & $n-\mathrm{Bu}$ & 85 & - & 70 \\
\hline 4 & $\mathrm{Ph}$ & $t-\mathrm{Bu}$ & - & - & 64 \\
\hline 5 & $3-\mathrm{MeOC}_{6} \mathrm{H}_{4}$ & $\mathrm{Bn}$ & 71 & 88 & - \\
\hline 6 & $3-\mathrm{MeOC}_{6} \mathrm{H}_{4}$ & $n-\mathrm{Bu}$ & 80 & 81 & - \\
\hline 7 & 2-Naphthyl & $\mathrm{Bn}$ & 73 & 55 & - \\
\hline 8 & 2-Naphthyl & $n-\mathrm{Bu}$ & 76 & 78 & - \\
\hline 9 & $4-\mathrm{MeOC}_{6} \mathrm{H}_{4}$ & $\mathrm{Bn}$ & 81 & 89 & 71 \\
\hline 10 & $4-\mathrm{MeOC}_{6} \mathrm{H}_{4}$ & $n-\mathrm{Bu}$ & 68 & 82 & 65 \\
\hline 11 & 4- $\mathrm{MeOC}_{6} \mathrm{H}_{4}$ & $t-\mathrm{Bu}$ & - & - & 56 \\
\hline 12 & 4- $\mathrm{MeOC}_{6} \mathrm{H}_{4}$ & $4-\mathrm{MeOC}_{6} \mathrm{H}_{4}$ & 80 & 80 & - \\
\hline 13 & 4- $\mathrm{NO}_{2} \mathrm{C}_{6} \mathrm{H}_{4}$ & $\mathrm{Bn}$ & 74 & 67 & 70 \\
\hline 14 & $4-\mathrm{NO}_{2} \mathrm{C}_{6} \mathrm{H}_{4}$ & $n-\mathrm{Bu}$ & 82 & 75 & 65 \\
\hline 15 & 2-Furyl & $\mathrm{Bn}$ & - & 68 & 74 \\
\hline
\end{tabular}

代丁二酰亚胺(NCS, NBS 和 NIS)等, 氯气和溴素作为较 廉价卤化试剂的缺点在于活性过高、均属于危险物质, 而 $N$-卤代丁二酰亚胺的优点在于反应专一性强, 但是 价格昂贵、制备与使用过程中原子经济性差. 基于仿生 合成的氧化卤化反应(oxidative halogenation)以其绿色环 保的特点备受人们关注, 尤其是采用 $\mathrm{O}_{2}, \mathrm{H}_{2} \mathrm{O}_{2}$ 等作为氧 化剂的氧化卤化反应 ${ }^{[55]}$ 被认为是一种极有前景的反应. 至今已有很多以 $\mathrm{UHP}, \mathrm{NaBO}_{3} \cdot 4 \mathrm{H}_{2} \mathrm{O}^{[56,57]}, 2 \mathrm{Na}_{2} \mathrm{CO}_{3}$ ・ $3 \mathrm{H}_{2} \mathrm{O}_{2}{ }^{[6]}$ 等固态化的 $\mathrm{H}_{2} \mathrm{O}_{2}$ 为氧化剂的氧化卤化反应见于 文献报道.

Paul 等 ${ }^{[58]}$ 报道了在硅胶固载的乙酸上以 $\mathrm{NaBr}$ 为溴 化试剂, UHP 为氧化剂, 并以微波为促进条件, 合成 $\alpha-$ 溴代脂肪酮的反应，只需 $40 \sim 120 \mathrm{~s}$ 即可以 $70 \% \sim 80 \%$ 的收率获得溴化产物.

Lee 等 $^{[59]}$ 报道了在离子液体 $[\mathrm{bmim}] \mathrm{BF}_{4}$ 中, 以 $\mathrm{AlCl}_{3} \cdot 6 \mathrm{H}_{2} \mathrm{O}$ 为氯化试剂和 UHP 为氧化剂, 从芳基酮 46 合成了 $\alpha$-氯代芳基酮 47 的反应, 在 $60{ }^{\circ} \mathrm{C}$ 下反应 $4 \sim 20$ $\mathrm{h}$, 收率在 $72 \% \sim 97 \%$ 之间. Lee 等 ${ }^{[60]}$ 还研究了在同种离
子液体中, 以 $N$-卤代丁二酰亚胺 (NXS) 为卤化试剂, UHP 为氧化剂, 合成 $\alpha$-卤代芳基酮(47,48)的反应，在 $60{ }^{\circ} \mathrm{C}$ 下反应 1 20 h, 氯代收率在 $68 \% \sim 98 \%$ 之间，溴 代收率在 74\% 97\%之间. 结果比较见表 8 .

表 8 离子液体中 NXS/UHP 体系对羰基化合物的 $\alpha$-卤化反应 Table $8 \alpha$-Halogenation of carbonyl compounds with NXS/ UHP in ionic liquid

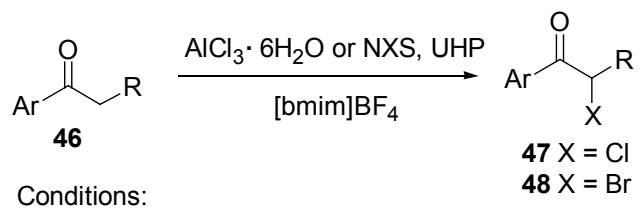

A: $\mathrm{AlCl}_{3} \cdot 6 \mathrm{H}_{2} \mathrm{O}$ (1.1 equiv.), UHP (2.0 equiv.), $60^{\circ} \mathrm{C}$ B: NXS ( 2.0 equiv.), UHP ( 1.2 equiv), $60{ }^{\circ} \mathrm{C}$

\begin{tabular}{|c|c|c|c|c|c|}
\hline \multirow{3}{*}{ Entry } & \multirow{3}{*}{$\mathrm{Ar}$} & \multirow{3}{*}{$\mathrm{R}$} & \multicolumn{3}{|c|}{ Yield/\% } \\
\hline & & & \multirow{2}{*}{$\mathrm{A}^{[59]}$} & \multicolumn{2}{|c|}{$\mathrm{B}^{[60]}$} \\
\hline & & & & $\mathrm{X}=\mathrm{Cl}$ & $\mathrm{X}=\mathrm{Br}$ \\
\hline 1 & $\mathrm{Ph}$ & $\mathrm{Me}$ & 77 & 78 & 76 \\
\hline 2 & $p-\mathrm{MeC}_{6} \mathrm{H}_{4}$ & $\mathrm{Me}$ & 72 & 76 & 86 \\
\hline 3 & $p-\mathrm{ClC}_{6} \mathrm{H}_{4}$ & $\mathrm{Me}$ & 77 & 76 & 77 \\
\hline 4 & $p-\mathrm{BrC}_{6} \mathrm{H}_{4}$ & $\mathrm{Me}$ & 72 & - & - \\
\hline 5 & $p-\mathrm{NO}_{2} \mathrm{C}_{6} \mathrm{H}_{4}$ & $\mathrm{Me}$ & - & 68 & 74 \\
\hline 6 & $\mathrm{Ph}$ & $\mathrm{Et}$ & 90 & 90 & 89 \\
\hline 7 & $\mathrm{Ph}$ & $n-\operatorname{Pr}$ & 88 & - & - \\
\hline 8 & $p-\mathrm{MeC}_{6} \mathrm{H}_{4}$ & Et & 94 & 70 & 78 \\
\hline 9 & $p-\mathrm{ClC}_{6} \mathrm{H}_{4}$ & Et & 80 & 94 & 76 \\
\hline 10 & $p-\mathrm{BrC}_{6} \mathrm{H}_{4}$ & Et & 97 & - & - \\
\hline 11 & $\mathrm{Ph}$ & $\mathrm{CH}_{2} \mathrm{COMe}$ & - & 78 & 96 \\
\hline 12 & $\mathrm{Ph}$ & $\mathrm{CH}_{2} \mathrm{CO}_{2} \mathrm{Et}$ & - & 95 & 97 \\
\hline 13 & & & 89 & 98 & 85 \\
\hline
\end{tabular}

最近, El-Ahl 等 ${ }^{[61]}$ 也报道了一种以 UHP 为氧化剂的 酮类 $\alpha$-氯代反应，但采用了有别于上述 $\mathrm{AlCl}_{3} \cdot 6 \mathrm{H}_{2} \mathrm{O}$ 和 $\mathrm{NXS}$ 的 $\mathrm{SiCl}_{4}(\mathrm{TCS})$ 作为氯化试剂. 酮类底物 49 在二氯 甲烷中 $0{ }^{\circ} \mathrm{C}$ 条件下反应 $1.0 \sim 1.5 \mathrm{~h}$, 可以 $76 \% \sim 93 \%$ 的 收率得到氯化产物 50 (Eq. 13). 实验中还发现, 当底物 为诸如二苯基乙酮、2,4-戊二酮等烯醇含量较高的酮类 物质时, 得到的是 $\alpha, \alpha$-二氯代产物, 且收率很高, 这可 能归因于羰基两侧的取代基使单氯代产物的烯醇式也 能较为稳定地存在, 从而容易发生二氯代反应. 作者还 尝试了以亚碘酰苯 $(\mathrm{PhIO})$ 为氧化剂进行反应, 但反应效 果与 UHP 相比较差, 反应时间长且收率低.

在氧化碘化反应中, $\mathrm{I}_{2} / \mathrm{UHP}$ 是常用的一种组合, 加 入微波等促进因素可显著提高反应. 由 Sosnowski 等 ${ }^{[62]}$ 报道的微波促进下氯仿中 $\mathrm{I}_{2} / \mathrm{UHP}$ 对苯胺 $(\mathbf{5 1})$ 的氧化碘 化反应(Eq. 14), 反应时间可以从常规反应条件下的 2 $3 \mathrm{~h}$ 缩短至 $10 \mathrm{~min}$, 但取代产物 52 的收率并不是很理想. 


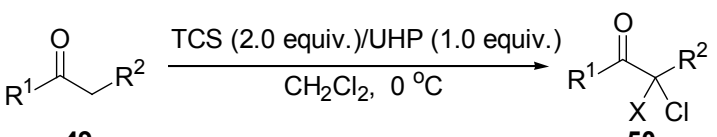

49

$\mathrm{R}^{1}=\mathrm{Ph}, \mathrm{R}^{2}=\mathrm{H}, \mathrm{X}=\mathrm{H}, 88 \%$;

$\mathrm{R}^{1}=4-\mathrm{MeC}_{6} \mathrm{H}_{4}, \mathrm{R}^{2}=\mathrm{H}, \mathrm{X}=\mathrm{H}, 92 \%$

$\mathrm{R}^{1}=4-\mathrm{MeOC}_{6} \mathrm{H}_{4}, \mathrm{R}^{2}=\mathrm{H}, \mathrm{X}=\mathrm{H}, 93 \%$;

$\mathrm{R}^{1}=4-\mathrm{BrC}_{6} \mathrm{H}_{4}, \mathrm{R}^{2}=\mathrm{H}, \mathrm{X}=\mathrm{H}, 92 \%$

$\mathrm{R}^{1}=4-\mathrm{ClC}_{6} \mathrm{H}_{4}, \mathrm{R}^{2}=\mathrm{H}, \mathrm{X}=\mathrm{H}, 93 \%$;

$\mathrm{R}^{1}=3-\mathrm{NO}_{2} \mathrm{C}_{6} \mathrm{H}_{4}, \mathrm{R}^{2}=\mathrm{H}, \mathrm{X}=\mathrm{H}, 85 \%$

$\mathrm{R}^{1}=$ 2-Naphthyl, $\mathrm{R}^{2}=\mathrm{H}, \mathrm{X}=\mathrm{Cl}, 87 \%$;

$\mathrm{R}^{1}=\mathrm{Ph}, \mathrm{R}^{2}=\mathrm{Ph}, \mathrm{X}=\mathrm{Cl}, 85 \%$

$\mathrm{R}^{1}=\mathrm{Me}, \mathrm{R}^{2}=\mathrm{MeCO}, \mathrm{X}=\mathrm{Cl}, 76 \%$

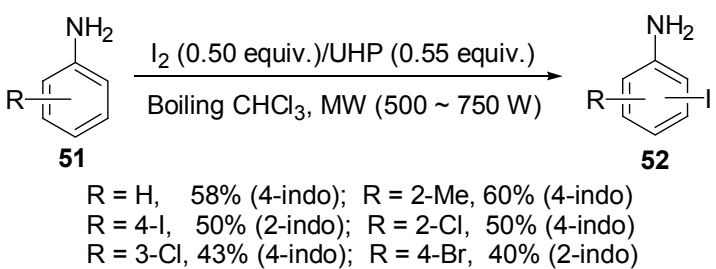

对于同属活化芳环的苯酚和苯甲醚及其衍生物, $\mathrm{I}_{2} / \mathrm{UHP}$ 体系同样适用. Pavlinac 等 ${ }^{[63]}$ 报道了以单质碘为 碘化试剂, UHP 为氧化剂, 无溶剂条件下实现在上述活 化芳环引入碘原子的反应(Eq. 15). 以对叔丁基苯酚(53) 为例, 所得碘化产物中有单取代 54 和二取代 55 两种, 原料的转化率及产物收率与 $\mathrm{I}_{2}$ 和 UHP 的投料量密切相 关, 实验还发现 UHP 的效果优于过碳酸钠 (SPC), 该结 论在作者类似的研究中也得到了佐证 ${ }^{[64]}$.

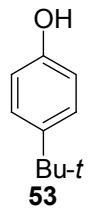

$$
\underset{\text { solvent-free }}{\stackrel{\mathrm{I}_{2}, \mathrm{UHP} \text { or SPC }}{\longrightarrow}}
$$
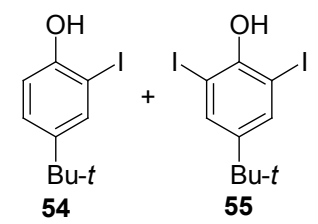

Conditions:

A: $I_{2}$ ( 0.5 equiv.), UHP ( 0.6 equiv.), $45^{\circ} \mathrm{C}, 5 \mathrm{~h}, 2$-iodo ( $50 \%$ ), 2,6-diiodo (25\%)

B: $\mathrm{I}_{2}$ (1.0 equiv.), UHP (1.0 equiv.), $45^{\circ} \mathrm{C}, 6 \mathrm{~h}, 2$-iodo ( $\left.30 \%\right)$, 2,6-diiodo (70\%)

C: $\mathrm{I}_{2}$ ( 0.5 equiv.), SPC ( 0.6 equiv.), $45^{\circ} \mathrm{C}, 6 \mathrm{~h}, 2$-iodo $(7 \%)$, 2,6-diiodo $(7 \%)$

D: $I_{2}$ (1.0 equiv.), UHP (1.0 equiv.), $45^{\circ} \mathrm{C}, 6 \mathrm{~h}, 2$-iodo (11\%), 2,6-diiodo (12\%)

对于钝化芳环 56 59 的氧化碘化, 常需要更加苛 刻的条件. Lulinski 等 ${ }^{[65]}$ 报道了一种以 $\mathrm{I}_{2} / \mathrm{UHP}$ 为碘化试 剂在 $\mathrm{Ac}_{2} \mathrm{O} / \mathrm{AcOH}$ 进行的反应(Eq. 16), 该系统的反应活 性由硫酸和 UHP 的添加量来调节.

氧化卤化反应, 特别是以 UHP 为氧化活化剂的反 应，具有原子经济性高的优势. 目前存在的主要问题是 在活化芳环上的卤化反应选择性较差, 而在钝化芳环上 的反应，尚需要加入其它的活化剂，反应结果也未达到

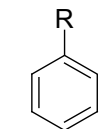

$56 \sim 59$

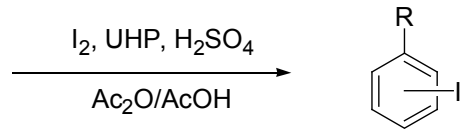

$60 \sim 63$
$56 \mathrm{R}=\mathrm{H}: \mathrm{I}_{2}$ (0.48 equiv.), UHP (0.71 equiv.), $\mathrm{H}_{2} \mathrm{SO}_{4}$ (19 equiv.), $45 \%$ $57 \mathrm{R}=\mathrm{Br}: \mathrm{I}_{2}$ (0.48 equiv.), UHP (0.71 equiv.), $\mathrm{H}_{2} \mathrm{SO}_{4}$ (19 equiv.), $83 \%$ (4-iodo)

$58 \mathrm{R}=\mathrm{COOH}: \mathrm{I}_{2}$ (0.55 equiv.), UHP (2.25 equiv.), $\mathrm{H}_{2} \mathrm{SO}_{4}$ (15 equiv.), $81 \%$ (3-iodo)

$59 \mathrm{R}=\mathrm{CF}_{3}: \mathrm{I}_{2}$ (0.55 equiv.), UHP (2.25 equiv.), $\mathrm{H}_{2} \mathrm{SO}_{4}$ (30 equiv.), $18 \%$ (3-iodo)

令人满意的程度. 因此开发能够增强 UHP 氧化活性、提 高卤化反应选择性且可循环使用的催化剂是改进该类 反应的关键所在.

\section{6 其他官能团的氧化反应}

除了上述提及的四大类反应外，还有几类有过氧化 嫝参与的氧化反应所涉及的实例较少, 如将在这部分中 集中讨论.

\section{1 水解腈成酰胺(Radziszewski 反应)}

腈类化合物的水解反应常用于相应的酰胺的制备, 该反应多采用强酸性条件, 如硫酸和盐酸, 且可以加入 金属、金属氧化物或金属络合物作为催化剂，但很少采 用碱性环境, 主要问题是所得到的产物酰胺会进一步水 解转化成羧酸(盐)造成收率下降. 1993 年 Balicki 等 ${ }^{[66]}$ 在 研究了 UHP 在大量有机反应中的应用后, 提出了在丙 酮一水混合溶剂中, 以 $\mathrm{K}_{2} \mathrm{CO}_{3}$ 为催化剂, 以过量的 UHP 为氧化剂室温下将腈类化合物 64 转化成相应的酰胺 65 的方法(Eq. 17), 反应时间 $0.5 \sim 2.5 \mathrm{~h}$, 收率在 $77 \%$ $96 \%$ 之间. 该方法表现出优异的选择性，当底物中含有 乙氧羰基(酯键)和甲酰胺基等对水敏感基团时反应也能 有较好的结果.

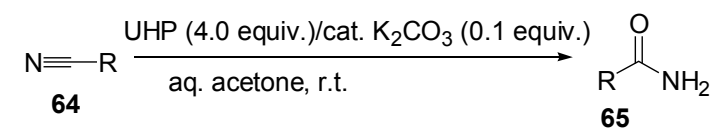

$\mathrm{R}=\mathrm{Ph}(85 \%), 4-\mathrm{ClC}_{6} \mathrm{H}_{4}(93 \%)$, 4-pyridyl (95\%), 3-pyridyl (95\%), $\mathrm{Bn}(90 \%), \mathrm{PhCO}(82 \%), 4-\mathrm{NO}_{2}$-2-pyridyl (94\%), 2-Cl-3-pyridyl (96\%), 6-MeO-3,4'-bipyridin-5-yl (80\%), 6-Cl-3,4'-bipyridin-5-yl (95\%), $\mathrm{Me}(77 \%)$, Et (80\%), $\mathrm{H}_{2} \mathrm{NCOCH}_{2}(90 \%), \mathrm{EtO}_{2} \mathrm{CCH}_{2}(95 \%), \mathrm{ClCH}_{2}$ $(87 \%)$.

\section{2 氧化芳香醛成芳香羧酸}

芳香醛转化成相应的羧酸常用的试剂有很多, 诸如 $\mathrm{KMnO}_{4}, \mathrm{CrO}_{3}$, 重铬酸吡啶(PDC), 三氧化铬吡啶盐酸 盐(PCC)等传统氧化剂在该反应中虽然应用较多, 但依 然存在收率低、价格高、污染大等问题，而采用 $\mathrm{H}_{2} \mathrm{O}_{2}$ 时反应相对环保, 但低浓度 $\mathrm{H}_{2} \mathrm{O}_{2}$ 溶液氧化性不够, 高浓 度的极易爆炸，在应用中存在较大的安全隐患. 2001 年 Balicki 等 ${ }^{[67]}$ 首先报道了采用 $\mathrm{UHP} / \mathrm{HCOOH}$ 这个体系将 
芳香醛(66)转化成芳香羧酸(67)的反应(Eq. 18), 在室温 下反应 $1.5 \sim 3 \mathrm{~h}$ 即可将芳香醛氧化成相应的芳香羊酸. 该方法的优势在于: (1)氧化剂安全稳定且价格低廉; (2) 反应条件温和、反应迅速、收率高、后处理简单; (3)该 方法在氧化 $N$-杂芳香醛的反应中同样具有高效率.

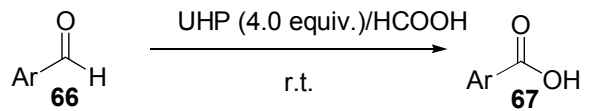

$\mathrm{Ar}=\mathrm{Ph}(96 \%), 2-\mathrm{NO}_{2} \mathrm{C}_{6} \mathrm{H}_{4}(79 \%), 3-\mathrm{NO}_{2} \mathrm{C}_{6} \mathrm{H}_{4}(78 \%), 2-\mathrm{ClC}_{6} \mathrm{H}_{4}(78 \%)$, 4- $-\mathrm{ClC}_{6} \mathrm{H}_{4}(82 \%), 3-\mathrm{ClC}_{6} \mathrm{H}_{4}(77 \%), 4-\mathrm{FC}_{6} \mathrm{H}_{4}(75 \%), 4-\mathrm{MeC}_{6} \mathrm{H}_{4}(85 \%)$, $3-\mathrm{MeC}_{6} \mathrm{H}_{4}(87 \%), 3-\mathrm{MeOC}_{6} \mathrm{H}_{4}(51 \%), 4-\mathrm{MeOC}_{6} \mathrm{H}_{4}(54 \%)$, 4-pyridyl (91\%), 3-pyridyl (90\%), 3-quinolyl (90\%)

同年 Heaney 等 ${ }^{[68]}$ 更系统地研究了 UHP 氧化甲氧基 取代的芳香醛得到相应的芳香羧酸的反应(Eq. 19). 针 对 MMPP 和 UHP- $\mathrm{Ac}_{2} \mathrm{O}$ 两个体系在甲氧基取代的芳香 醛氧化反应中, 醛基氧化成羧基与 Baeyer-Villiger (Dakin)反应并存的问题, 指出在碱性条件下将反应中四取 代的 Criegee 中间体将更容易消除一个质子, 该步反应 将快于芳香基的迁移，从而抑制 Baeyer-Villiger (Dakin) 反应, 主产物将是芳香羧酸, 实验结果也确实验证了这 一说法. 反应在室温下甲醇中进行, 加入高浓度的 $\mathrm{NaOH}$ 溶液和 UHP 固体, 再加热至 $65{ }^{\circ} \mathrm{C}$, 即可以较高 至很高的收率得到产物芳香羒酸.

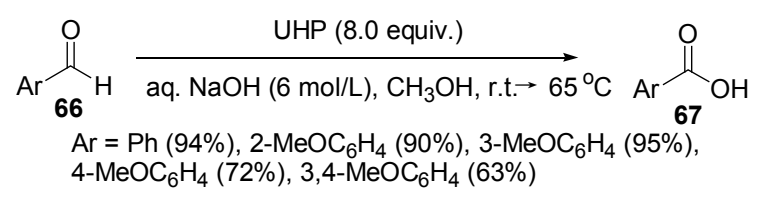

\section{3 氧化羟基成羰基}

将醇类化合物氧化成相应的醛或酮是有机合成中 重要的官能团转化反应之一. 该反应中所用的氧化剂有 很多, 常见的有 $\mathrm{KMnO}_{4}, \mathrm{RuO}_{4}$, 二吡啶合三氧化铬 (Collins 试剂), 重铬酸吡定(PDC), 三氧化铬吡定盐酸盐 (PCC)等, 这些试剂的优点在于往往具有高度的选择性 和高产率, 但是反应产生大量的重金属废弃物, 给反应 的后处理也带来了极大的麻烦. 2009 年 Park 等 ${ }^{[69]}$ 报道了 在离子液体 $[\mathrm{bmim}] \mathrm{BF}_{4}$ 中, 以 $\mathrm{MgBr}_{2}$ 为催化剂, UHP 氧 化伯醇 68 与仲醇 69 分别生成对应的醛 70 和酮 71 的反 应(Eq. 20). 在 $60{ }^{\circ} \mathrm{C}$ 的温度下反应 $2 \sim 3 \mathrm{~h}$, 产物收率在 75\% 94\%之间. 该方法虽然尚未推广到更多的化合物, 其普适性尚待考证, 但它所具有的环境友好的特点值得 关注.

\section{4 氧化肜成硝基化合物}

硝基在有机合成中是一个很有用的基团，可以通过 各种反应转化成其他一系列的官能团. 脂肪硝基化合物

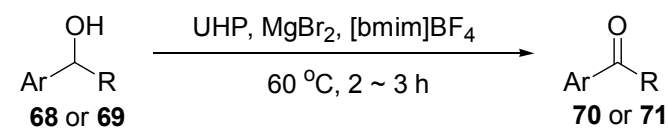

primary alcohol $68 \rightarrow 70$

$\mathrm{R}=\mathrm{H} ; \mathrm{Ar}=\mathrm{Ph}(94 \%), 4-\mathrm{MeC}_{6} \mathrm{H}_{4}(92 \%), 4-\mathrm{FC}_{6} \mathrm{H}_{4}(88 \%)$, $4-\mathrm{ClC}_{6} \mathrm{H}_{4}(87 \%), 4-\mathrm{BrC}_{6} \mathrm{H}_{4}(85 \%), 4-\mathrm{MeOC}_{6} \mathrm{H}_{4}(91 \%)$, 4- $\mathrm{NO}_{2} \mathrm{C}_{6} \mathrm{H}_{4}(77 \%), 4-\mathrm{CF}_{3} \mathrm{C}_{6} \mathrm{H}_{4}(78 \%), 2$-naphthyl $(75 \%)$

secondary alcohol $69 \rightarrow 71$

$\mathrm{R}=\mathrm{Me} ; \mathrm{Ar}=\mathrm{Ph}(91 \%), 4-\mathrm{MeC}_{6} \mathrm{H}_{4}(90 \%), 4-\mathrm{FC}_{6} \mathrm{H}_{4}(85 \%)$, $4-\mathrm{ClC}_{6} \mathrm{H}_{4}(84 \%), 4-\mathrm{BrC}_{6} \mathrm{H}_{4}(84 \%)$;

$\mathrm{Ar}=\mathrm{Ph} ; \mathrm{R}=\mathrm{Et}(86 \%), \mathrm{Ph}(87 \%), \mathrm{COPh}(87 \%) \mathrm{CO}_{2} \mathrm{Me}(78 \%)$ $\mathrm{CO}_{2} \mathrm{Et}(78 \%)$

的制备方法主要有两种: (1)伯或仲溴化物或磑化物与亚 硝酸钠或亚硝酸银反应，反应的主要副产物是亚硝酸 酯; (2)将伯胺、䏡、叠氮化物、异氧酸酯等化合物氧化 成硝基化合物, 常用的氧化剂有干燥的 $\mathrm{O}_{3}$, 各类过酸, $\mathrm{MTO} / \mathrm{H}_{2} \mathrm{O}_{2}$, Oxone, 过硼酸钠等.

1992 年 Ballini 等 ${ }^{[70]}$ 首次报道了以 UHP/TFAA 体系 氧化肜 72 合成硝基化合物 73 的反应(表 9). 该反应中, 以乙腈为溶剂, 在 $0{ }^{\circ} \mathrm{C}$ 下底物肜均能以很好的收率得 到硝基化合物. 值得一提的是, 当肜基的 $\alpha$ 位上具有手 性中心时, 在较高转化率的前提下, 化合物的原有构型 保持不变. 但该方法的主要问题是 UHP 与 TFAA 需要 大大过量，也造成了反应的选择性并不理想.

表 $9 \mathrm{UHP} / \mathrm{TFAA}$ 体系氧化肜合成硝基化合物的反应 Table 9 Oxidation of aldoximes to nitroalkanes with UHP/TFAA

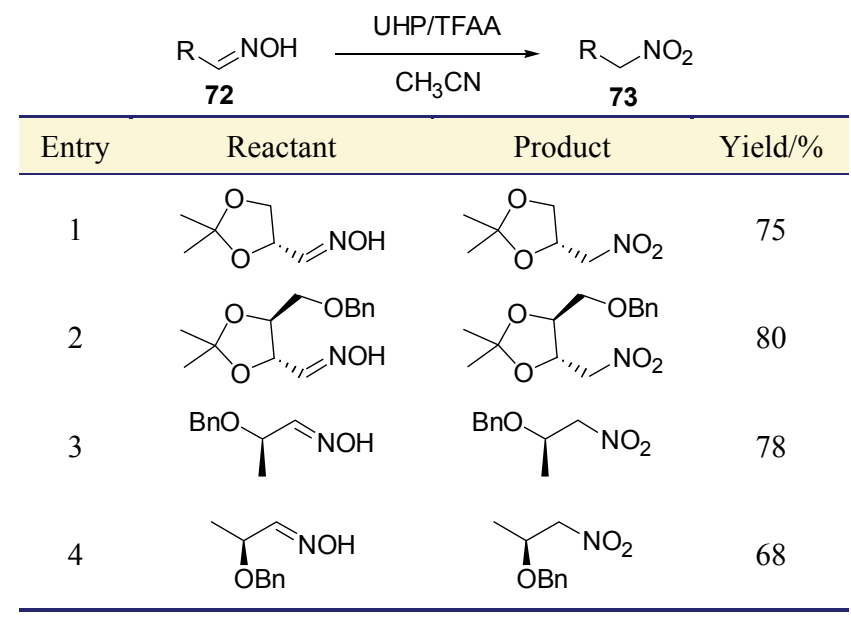

2004 年 Cardona 等 ${ }^{[71]}$ 提出了一种在 MTO 催化下用 UHP 氧化芳香肪 74 生成相应的硝基化合物 73 的反应 (Eq. 21), 可显著地降低氧化剂的用量. 反应在室温下进 行, 以甲醇或乙腈为溶剂, UHP 用量为 3 倍, 催化剂 MTO 用量为 2 $10 \mathrm{~mol} \%$, 柱色谱得到目标产物. 但这 一方法并不适用于脂肪醛肟的氧化，作者尝试了正庚醛 肟的氧化，结果只得到复杂的混合物. 


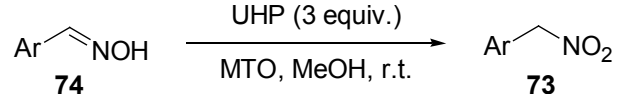

$\mathrm{Ar}=\mathrm{Ph}(50 \%), \mathrm{Ar}=4-\mathrm{MeOC}_{6} \mathrm{H}_{4}(46 \%), \mathrm{Ar}=2-\mathrm{NO}_{2} \mathrm{C}_{6} \mathrm{H}_{4}(35 \%)$

\section{5 氧化呋喃开环}

呋喃环的开环反应得到不饱和的 1,4-二羰基化合 物, 后者可以是马来醛这样的不稳定化合物, 在不分离 的条件下即可参与下一步合成, 因此在合成反应中有较 大的意义. 1998 年 Finlay 等 ${ }^{[72]}$ 报道了在 MTO 的催化下 呋喃类化合物 75 与 UHP 作用, 氧化开环生成不饱和 1,4-二羰基化合物 76 (Eq. 22), 收率在 67\% 97\%之间， 副产物尿素经过滤即可除去, 产物经简单的快速柱色谱 即可提纯.

$$
\begin{aligned}
& \mathrm{R}^{1}=\mathrm{H}, \mathrm{Me}, \mathrm{Et}, \mathrm{Ph}, \mathrm{CH}_{2} \mathrm{OCO}\left(\mathrm{CH}_{2}\right)_{12} \mathrm{CH}_{3} \\
& \mathrm{R}^{2}=\mathrm{Me}, \mathrm{Et}, \mathrm{Ph}, \mathrm{OMe}, \mathrm{CH}_{2} \mathrm{OAc}, \mathrm{CH}(\mathrm{Me}) \mathrm{OAc}, \mathrm{CH}(\mathrm{Et}) \mathrm{OAc} \\
& \mathrm{R}^{3}=\mathrm{H}, \mathrm{COMe}, \mathrm{CO}_{2} \mathrm{Et}
\end{aligned}
$$

\section{6 氧化芳构化}

1,4 -二氢吡啶 $(1,4-\mathrm{DHP})$ 氧化成相应的吡啶是 1,4-DHP 类药物的主要代谢途径, 因此研究 1,4-DHP 的 氧化芳构化有助于研究上述生物转化过程, 同时也是一 种多取代吡啶的合成方法. 该反应常用的氧化剂有硝 酸、亚硝酸、硝酸盐等氧化剂, 但这些氧化剂往往具有 选择性差、操作繁琐以及产生大量有毒有害废水的缺陷. 而将 UHP 用于 1,4-DHP 的氧化芳构化反应, 可极大地 回避上述缺陷. 2008 年 Filipan-Litvic 等 ${ }^{[73]}$ 报道了以 UHP 为氧化剂, 碘为催化剂将 1,4-DHP (77)芳构化的反应 (Eq. 23), 反应在室温下进行, 从众多溶剂中篮选出了最 佳溶剂乙酸乙酯，反应收率在 $73 \% ～ 99 \%$ 之间.

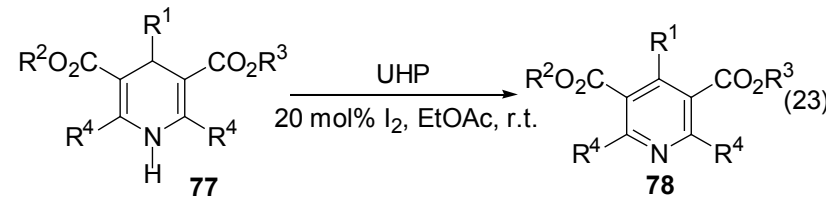

$\mathrm{R}^{1}=\mathrm{H}, i-\mathrm{Pr}, \mathrm{CH}_{2} \mathrm{Ph}, \mathrm{Et}, n-\mathrm{Pr}, \mathrm{Ph}, 2-\mathrm{ClC}_{6} \mathrm{H}_{4}, 3-\mathrm{ClC}_{6} \mathrm{H}_{4}, 4-\mathrm{ClC}_{6} \mathrm{H}_{4}$, 2- $-\mathrm{NO}_{2} \mathrm{C}_{6} \mathrm{H}_{4}, 3-\mathrm{NO}_{2} \mathrm{C}_{6} \mathrm{H}_{4}, 4-\mathrm{NO}_{2} \mathrm{C}_{6} \mathrm{H}_{4}, 2-\mathrm{MeC}_{6} \mathrm{H}_{4}, 3-\mathrm{MeC}_{6} \mathrm{H}_{4}$, 4- $\mathrm{MeC}_{6} \mathrm{H}_{4}, 2,4-\mathrm{Me}_{2} \mathrm{C}_{6} \mathrm{H}_{3}, 2-\mathrm{MeOC}_{6} \mathrm{H}_{4}, 3-\mathrm{MeOC}_{6} \mathrm{H}_{4}, 4-\mathrm{MeOC}_{6} \mathrm{H}_{4}$, 2-thienyl, 2-(5-Br-thienyl), 2-furyl, 4-Ph- $\mathrm{C}_{6} \mathrm{H}_{4}$ $\mathrm{R}^{2}=\mathrm{Me}, \mathrm{Et}, i-\mathrm{Pr}, t-\mathrm{Bu} ; \mathrm{R}^{3}=\mathrm{Me}, \mathrm{Et}, i-\mathrm{Pr}, t-\mathrm{Bu} ; \mathrm{R}^{4}=\mathrm{Me}, n-\mathrm{Pr}$

\section{7 醛的氧化酰胺化}

2008 年 Suto 等 ${ }^{[74]}$ 报道了在 $\mathrm{PdCl}_{2}$-xantophos 催化下, 以 UHP 为氧化剂, 醛 79 与胺 80 的氧化酰胺化反应(Eq. 24). 作者最初尝试了 $\mathrm{H}_{2} \mathrm{O}_{2}$ 水溶液为氧化剂的苯甲醛氧 化酰胺化反应, 在不加入催化剂的条件下, 酰胺的收率
为 $26 \%$, 若以 $\mathrm{Pd}(\mathrm{OAc})_{2}$ 为催化剂收率可至 $47 \%$, 主要的 副产物是苯甲酸, 后者由苯甲醛的直接氧化形成, 作者 推测 $\mathrm{H}_{2} \mathrm{O}_{2}$ 水溶液中的水阻碍了反应中关键中间体亚胺 的形成，因此作者用 UHP 替换了 $\mathrm{H}_{2} \mathrm{O}_{2}$ 溶液，结果苯甲 酸的含量显著下降. 而换用其他的氧化剂, 诸如 TBHP, Oxone 和 $m$-CPBA 后, 效果均很差. 在众多配体中选定 了 4,5-双二苯基膦-9,9-二甲基氧杂葱(xantphos). 最终, 一系列醛类和胺类，在 $\mathrm{MeOH} / \mathrm{AcOH}$ 溶剂体系中, $50{ }^{\circ} \mathrm{C}$ 下反应 $20 \mathrm{~h}$, 得到相应的酰胺 81, 收率在 13\% 86\%之 间。

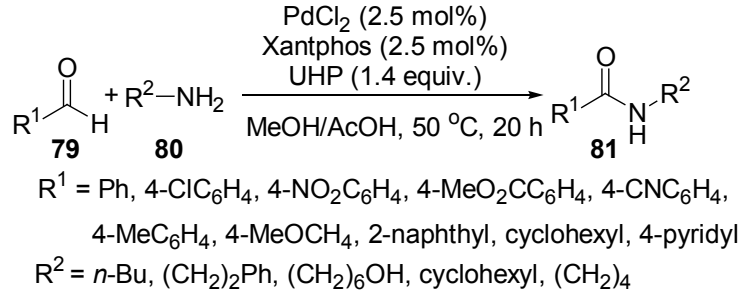

\section{7 结语}

过氧化腿的制备方法提出已有 70 年，它在日化、纺 织、卫生等领域已有广泛的应用，但作为一种氧化剂在 有机反应得到应用，只有短短二十余年. 目前，过氧化 艮作为 $\mathrm{H}_{2} \mathrm{O}_{2}$ 溶液和有机过酸的替代物已经在众多反应 中得到了应用, 并且有很好的效果. 随着绿色化学和安 全生产的观念不断地深入人心, 过氧化艮在氧化反应中 有着较好的应用前景, 对工业化生产也有深远的影响.

在现有的反应中，过氧化脲与酸酐的组合虽然在很 多反应中取得了较好的结果，但从反应机理上分析，真 正发挥氧化剂作用的是原位生成的过氧酸, 这必然造成 了过氧酸氧化反应的缺陷依然存在. 此外, 为了达到反 应所要求的氧化能力, 往往需要过量使用过氧化嫝, 这 造成了反应选择性的下降. 寻找更高催化活性、价格更 低廉和方便反应后处理的催化剂, 使得过氧化脲具有更 高的反应活性和选择性，在温和的条件下发挥过氧化脲 的 $\mathrm{H}_{2} \mathrm{O}_{2}$ 缓释功能这一优势, 获得更好的反应效果是当 前亟待解决的问题，含氟质子性溶剂与过氧化豚的结合 使用是一条较有前景的途径.

\section{References}

[1] Jones, C. W. Applications of Hydrogen Peroxide and Derivatives, Royal Society of Chemistry, Cambridge, 1999, p. 79.

[2] Lane, B. S.; Burgess, K. Chem. Rev. 2003, 103(7), 2457.

[3] Sato, K.; Aoki, M.; Noyori, R. Science 1998, 281(5383), 1646.

[4] Baxendale, J. H.; Wilson, J. A. Trans. Faraday Soc. 1957, 53, 344.

[5] Mckillop, A.; Sanderson, W. R. Tetrahedron 1995, 51(22), 6145.

[6] Muzart, J. Synthesis 1995, 1325.

[7] McKillop, A.; Sanderson, W. R. J. Chem. Soc., Perkin Trans. 1 2000, (4), 471 
[8] Cooper, M. S.; Heaney, H.; Newbold, A. J.; Sanderson, W. R. Synlett 1990, (9), 533.

[9] Armarego, W. L. F.; Chai, C. L. L. Purification of Laboratory Chemicals, 6th ed., Elsevier, Oxford, 2009, p. 470.

[10] Gonsalves, A. M. D. R.; Johnstone, R. A. W.; Pereira, M. M.; Shaw, J. J. Chem. Res., Synop. 1991, (8), 208.

[11] Smith, M. B.; March, J. Advanced Organic Chemistry: Reactions Mechanisms Structure, John Wiley \& Sons, New Jersey, 2007, p. 1169.

[12] Adam, W.; Mitchell, C. M. Angew. Chem., Int. Ed. 1996, 35(5), 533.

[13] Boehlow, T. R.; Spilling, C. D. Tetrahedron Lett. 1996, 37(16), 2717.

[14] Malhotra, R.; Kumar, S.; Satyam, A. J. Chem. Soc., Chem. Commun. 1994, (11), 1339.

[15] Balch, A. L.; Costa, D. A.; Noll, B. C.; Olmstead, M. M. J. Am. Chem. Soc. 1995, 117(35), 8926.

[16] Murray, R. W.; Iyanar, K. Tetrahedron Lett. 1997, 38(3), 335.

[17] Legros, J.; Crousse, B.; Bonnet-Delpon, D.; Begue, J. P. Eur. J. Org. Chem. 2002, (19), 3290.

[18] Fan, C. L.; Lee, W. D.; Teng, N. W.; Sun, Y. C.; Chen, K. M. J. Org. Chem. 2003, 68(25), 9816.

[19] Valderrama, J. A.; Gonzalez, M. F.; Torres, C. Heterocycles 2003, 60(10), 2343.

[20] Ankudey, E. G.; Olivo, H. F.; Peeples, T. L. Green Chem. 2006, $8(10), 923$.

[21] Jin, H.; Zhao, H. Y.; Zhao, F. H.; Li, S. H.; Liu, W.; Zhou, G. P.; Tao, K.; Hou, T. P. Ultrason. Sonochem. 2009, $16(3), 304$.

[22] Mahmoodi, N. O.; Yazdanbakhsh, M. R.; Ghanbari, F. Synth. Commun. 2010, 40(21), 3181.

[23] Bouh, A. O.; Espenson, J. H. J. Mol. Catal. A: Chem. 2003, $200(1 \sim 2), 43$.

[24] Ichihara, J.; Iteya, K.; Kambara, A.; Sasaki, Y. Catal. Today 2003, $87(1 \sim 4), 163$.

[25] Saladino, R.; Bernini, R.; Neri, V.; Crestini, C. Appl. Catal. A 2009, $360(2), 171$.

[26] Carreiro, E. D.; Burke, A. J.; Curto, M. J. M.; Teixeira, A. J. J. Mol. Catal. A: Chem. 2004, 217(1 2), 69.

[27] Kureshy, R. I.; Khan, N. U. H.; Abdi, S. H. R.; Singh, S.; Ahmed, I.; Shukla, R. S.; Jasra, R. V. J. Catal. 2003, $219(1), 1$.

[28] Carreiro, E. D.; Yong-En, G.; Burke, A. J. J. Mol. Catal. A: Chem. 2005, 235(1 2), 285.

[29] Egami, H.; Katsuki, T. Angew. Chem., Int. Ed. 2008, 47(28), 5171.

[30] Egami, H.; Oguma, T.; Katsuki, T. J. Am. Chem. Soc. 2010, 132(16), 5886.

[31] Maity, N. C.; Abdi, S. H. R.; Kureshy, R. I.; Khan, N. U. H.; Suresh, E.; Dangi, G. P.; Bajaj, H. C. J. Catal. 2011, $277(1), 123$.

[32] Marigo, M.; Bachmann, S.; Halland, N.; Braunton, A.; Jorgensen, K. A. Angew. Chem., Int. Ed. 2004, 43(41), 5507.

[33] Rios, M. Y.; Salazar, E.; Olivo, H. F. Green Chem. 2007, 9(5), 459.

[34] Marcos, I. S.; Beneitez, A.; Castaneda, L.; Moro, R. F.; Basabe, P.; Diez, D.; Urones, J. G. Synlett 2007, (10), 1589.

[35] Garrido, N. M.; Garcia, M.; Sanchez, M. R.; Diez, D.; Urones, J. G. Synlett 2010, (3), 387.

[36] Gunaratne, H. Q. N.; McKervey, M. A.; Feutren, S.; Finlay, J.; Boyd, J. Tetrahedron Lett., 1998, 39(31), 5655.

[37] Balicki, R. J. Prakt. Chem., 1999, 341(2), 184.

[38] Balicki, R. Synth. Commun. 1999, 29(13), 2235.
[39] Varma, R. S.; Naicker, K. P. Org. Lett. 1999, 1(2), 189.

[40] Sasaki, Y.; Ushimaru, K.; Iteya, K.; Nakayama, H.; Yamaguchi, S.; Ichihara, J. Tetrahedron Lett. 2004, 45(52), 9513.

[41] Hasaninejad, A.; Chehardoli, G.; Zolfigol, M. A.; Abdoli, A. Phosphorus, Sulfur Silicon Relat. Elem. 2011, 186(2), 271.

[42] Saito, B.; Katsuki, T. Tetrahedron Lett. 2001, 42 (23), 3873.

[43] Miyazaki, T.; Katsuki, T. Synlett 2003, (7), 1046.

[44] Bernier, D.; Wefelscheid, U. K.; Woodward, S. Org. Prep. Proced. Int. 2009, 41(3), 173.

[45] Kaczmarek, L.; Balicki, R.; Nantkanamirski, P. Chem. Ber. 1992, 125(8), 1965.

[46] Caron, S.; Do, N. M.; Sieser, J. E. Tetrahedron Lett. 2000, 41(14), 2299.

[47] Balicki, R.; Golinski, J. Synth. Commun. 2000, 30(8), 1529.

[48] Rong, D. W.; Phillips, V. A.; Rubio, R. S.; Castro, M. A.; Wheelhouse, R. T. Tetrahedron Lett., 2008, 49(48), 6933.

[49] Marcantoni, E.; Petrini, M.; Polimanti, O. Tetrahedron Lett. 1995, $36(20), 3561$.

[50] Goti, A.; Nannelli, L. Tetrahedron Lett. 1996, 37(33), 6025.

[51] Murray, R. W.; Iyanar, K.; Chen, J. X.; Wearing, J. T. J. Org. Chem. 1996, 61(23), 8099.

[52] Soldaini, G.; Cardona, F.; Goti, A. Org. Lett. 2007, 9(3), 473.

[53] Cardona, F.; Bonanni, M.; Soldaini, G.; Goti, A. ChemSusChem 2008, 1(4), 327.

[54] Singh, B.; Jain, S. L.; Khatri, P. K.; Sain, B. Green Chem. 2009, 11(12), 1941.

[55] Podgoršek, A.; Zupan, M.; Iskra, J. Angew. Chem., Int. Ed. 2009, 48(45), 8424.

[56] Roche, D.; Prasad, K.; Repic, O.; Blacklock, T. J. Tetrahedron Lett. 2000, 4l(13), 2083.

[57] Kabalka, G. W.; Yang, K. Synth. Commun. 1998, 28(20), 3807.

[58] Paul, S.; Nanda, P.; Gupta, R. Indian J. Chem., Sect. B: Org. Chem. Incl. Med. Chem. 2005, 44(1), 184.

[59] Lee, J. C.; Park, H. J. Synth. Commun. 2006, 36(6), 777.

[60] Lee, J. C.; Park, H. J. Synth. Commun. 2007, 37(1-3), 87.

[61] El-Ahl, A. A. S.; Elbeheery, A. H.; Amer, F. A. Synth. Commun. 2011, 41(10), 1508.

[62] Sosnowski, M.; Skulski, L. Molecules 2002, 7(12), 867.

[63] Pavlinac, J.; Zupan, M.; Stavber, S. Org. Biomol. Chem. 2007, 5(4), 699.

[64] Pavlinac, J.; Zupan, M.; Stavber, S. Acta Chim. Slov. 2008, 55(4), 841.

[65] Lulinski, P.; Kryska, A.; Sosnowski, M.; Skulski, L. Synthesis 2004, (3), 441.

[66] Balicki, R.; Kaczmarek, L. Synth. Commun. 1993, 23(22), 3149.

[67] Balicki, R. Synth. Commun. 2001, 31(14), 2195.

[68] Heaney, H.; Newbold, A. J. Tetrahedron Lett. 2001, 42(37), 6607.

[69] Park, H. J.; Lee, J. C. Synlett 2009, (1), 79.

[70] Ballini, R.; Marcantoni, E.; Petrini, M. Tetrahedron Lett. 1992, 33(33), 4835.

[71] Cardona, F.; Soldaini, G.; Goti, A. Synlett 2004, (9), 1553.

[72] Finlay, J.; McKervey, M. A.; Gunaratne, H. Q. N. Tetrahedron Lett. 1998, 39(31), 5651.

[73] Filipan-Litvic, M.; Litvic, M.; Vinkovic, V. Tetrahedron 2008, 64(24), 5649.

[74] Suto, Y.; Yamagiwa, N.; Torisawa, Y. Tetrahedron Lett. 2008, 49(40), 5732. 